\title{
Synthesis and properties of conjugated phenylene ethynylene derivatives
}

\author{
Haiying Ji, Guohua Gao* \\ Shanghai Key Laboratory of Green Chemistry and Chemical Processes, Department of Chemistry, \\ East China Normal University, 3663 North Zhongshan Road, Shanghai 200062, China \\ E-mail:ghgao@chem.ecnu.edu.cn
}

\begin{abstract}
Fourteen new conjugated phenylene ethynylene derivatives as potential molecular electronic devices are presented here. These new compounds were synthesized by convergent synthetic methodologies which are based on Sonogashira and Suzuki couplings. The synthesized compounds were identified by ${ }^{1} \mathrm{H}$ NMR, ${ }^{13} \mathrm{C}$ NMR, IR, HRMS and elemental analysis. The $\mathrm{UV}$-vis maximum absorption spectra were in the range of 304-321 nm in $\mathrm{CHCl}_{3}$. $\mathrm{Cyclic}$ voltammetry $(\mathrm{CV})$ test results showed two distinct reduction peaks.
\end{abstract}

Keywords: Suzuki reaction, Sonogashira reaction, spectra properties, electrochemical properties

\section{Introduction}

In the past few years, molecular electronics has attracted much interest of chemists and physicists. ${ }^{1}$ Rigid rod conjugated oligo(phenylene ethynylene)s (OPEs) are one family of molecules that have interesting molecular electronic properties. ${ }^{2}$ OPEs derivatives containing thiol groups can form self-assembled monolayers (SAMs) on the surface of Au, Ag or other metals. ${ }^{3}$ SAMs plays an important role in the constructure ordered nanostructure in molecular electronics. Chemical functionalization of OPEs with nitro groups and terminal sulfur groups exhibits negative differential resistance (NDR) and molecular random access memory effects. ${ }^{4}$ Numerous theoretical studies of the nitro group effect and electron transport behavior are still under investigation. ${ }^{5}$ Several groups ${ }^{6}$ have been pursuing the ultimate miniaturization of electronic components, synthesizing diverse organic molecules that can be used as electronic devices. Since then, considerable work has been focused on their synthesis. ${ }^{7}$

Here, new conjugated phenylene ethynylene derivatives containing protected thiol end 
groups are designed and synthesized. These new compounds could be used as potential molecular electronics devices. Target compounds are synthesized using convergent synthetic methodologies which are based on Sonogashira ${ }^{8}$ and Suzuki ${ }^{9}$ couplings for aryl-ethynyl and aryl-aryl bond formations. In addition to these syntheses, the spectroscopic and electrochemical properties of these compounds were also tested.

\section{Results and Discussion}

\section{Synthesis}

The synthetic route of conjugated phenylene ethynylene derivatives $\mathbf{5}$ is outlined in Scheme 1. The central core of 4-bromo-1-iodo-2-nitrobenzene was synthesized according to the literature methods. ${ }^{7 \mathrm{~g}}$ Compound 1 was synthesized according to the literature methods ${ }^{7 \mathrm{~g}, 10}$ in $91 \%$ yields. Then compound $\mathbf{1}$ reacted with substituted phenylboronic acid to furnish compounds $\mathbf{2}$ in moderate $(17-84 \%)$ yields.
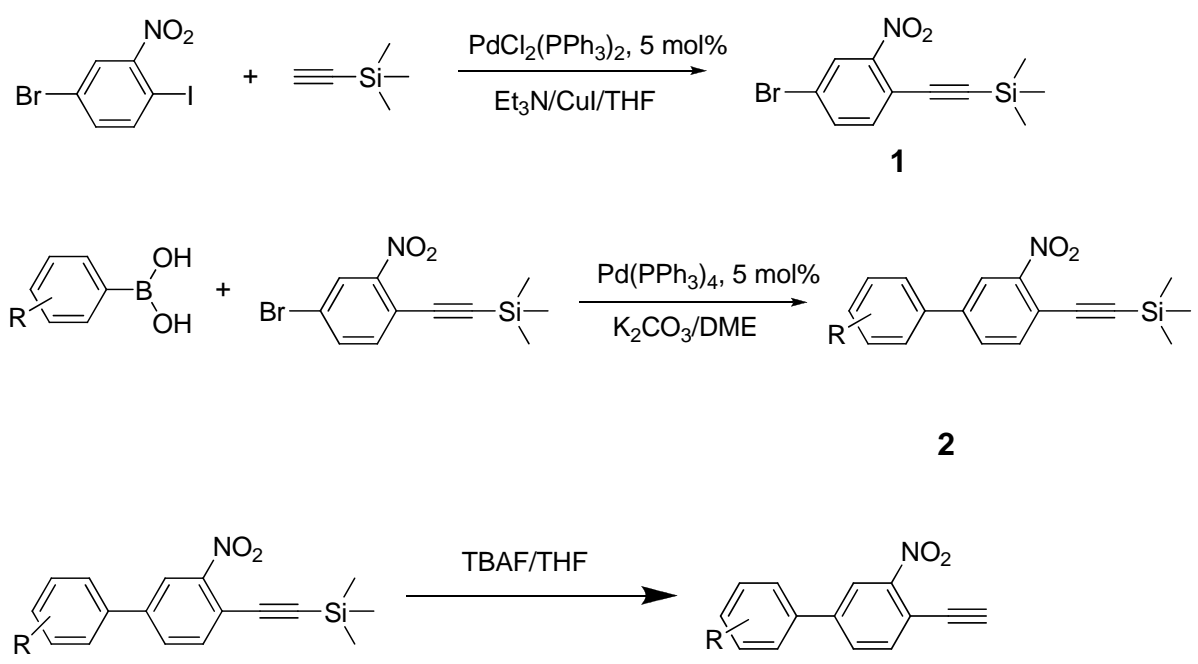

2

3

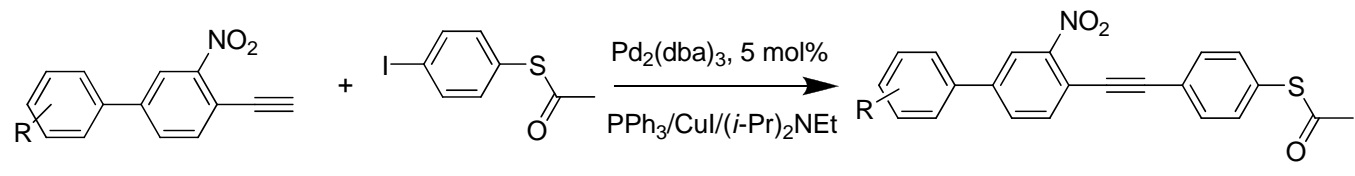

3

4

5

Sheme 1. The synthetic route of target compounds $\mathbf{5}$.

Synthesis of compounds 2 was carried out using 5 mol \% tetrakis(triphenylphosphine) 
palladium $(0)$ as catalyst and potassium carbonate $\left(\mathrm{K}_{2} \mathrm{CO}_{3}\right)$ as base in 1,2-dimethoxyethane (DME) and $\mathrm{H}_{2} \mathrm{O}$ at reflux temperature. Terminal alkyne $\mathbf{3 h}$ was also achieved in this step when $\mathrm{R}$ was 4-acetyl group and the yield was $31 \%$.

Sequentially, the compounds 2 were desilylated using tetrabutylammonium fluoride (TBAF) to furnish the terminal alkynes 3 in moderate to high (31-97\%) yields. Finally, conjugated phenylene ethynylene derivatives 5 can readily be achieved by reaction of the corresponding terminal alkynes 3 with the protected-thiol "alligator clip" $\mathbf{4}^{7 \mathrm{a}, 11}$ using palladium catalyzed Sonogashira cross coupling reaction. Reactions was carried out using tris(dibenzylideneacetone)dipalladium(0) (5 mol\% based on aryl halide) as catalyst, copper(I) iodide (10 mol\% based on aryl halide) as co-catalyst and triphenyl phosphine (20 mol\% based on aryl halide) as ligand in tetrahydrofuran at $50{ }^{\circ} \mathrm{C}$ with $N, N$-diisopropylethylamine as base. The structure and isolated yields of products $\mathbf{5}$ are shown in Table 1. The yields of product $\mathbf{5}$ are from 12 to $79 \%$. It is found that the yields were the highest when R was 4-thiomethyl group $\mathbf{5 m}$.

Table 1. Data of conjugated phenylene ethynylene derivatives 5

Compounds


Table 1. Continued

Compounds Yields (\%)

Variation of functional groups, both number and location can have a profound influence on the electronic properties of the compounds. ${ }^{12}$ Considering this, the derivatives 9 was prepared using a protocol similar to that described above.

4-Bromo-1-iodo-2-nitrobenzene was highly selectively coupled with 4-(methylthio)phenyl boronic acid to give cross-coupling product of compound 6 in $87 \%$ yield. Next 6 was coupled with trimethylsilylacetylene (TMSA) to afford 7, and then deprotected with TBAF to furnish 8. A final coupling with the protected-thiol "alligator clip" 4 afforded the desired product 9 in $45 \%$ yields. 


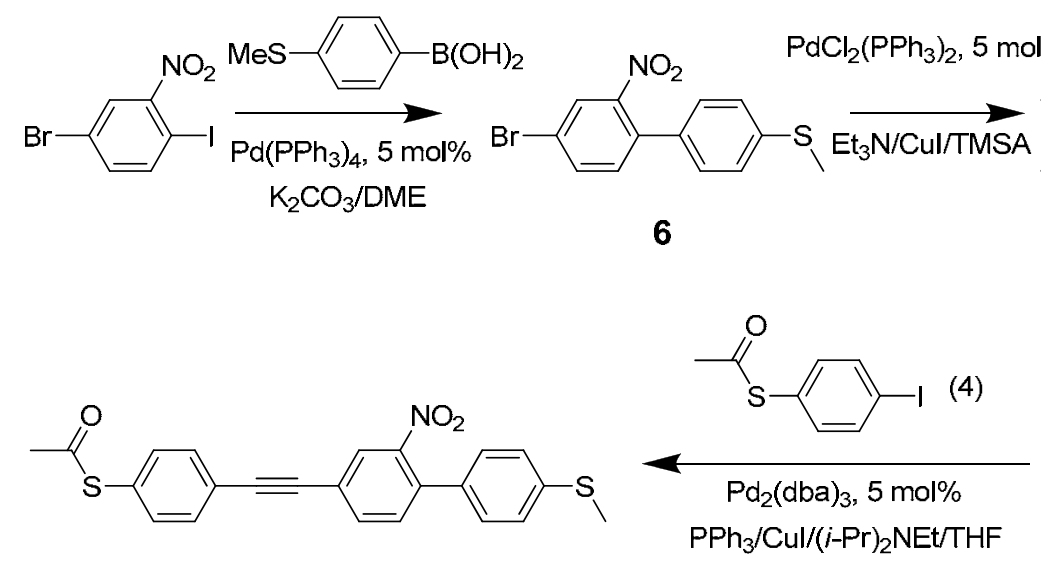

9
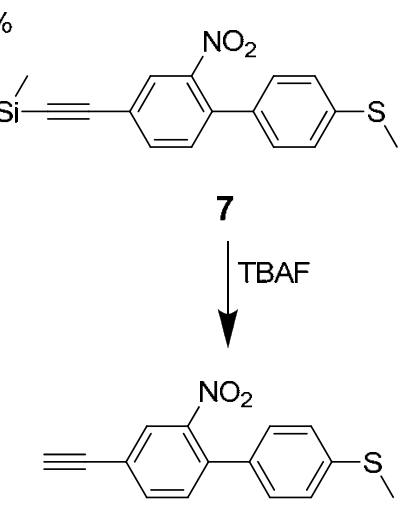

8

Scheme 2. The synthetic route to target compound 9.

\section{UV-vis spectral properties}

In order to study the optical properties of these compounds, the UV-vis absorption spectra were tested in $\mathrm{CHCl}_{3}$ solution. The UV-vis maximum absorption wavelength $\left(\lambda_{\mathrm{abs}}{ }_{\text {max }}\right)$ and molar absorption coefficient $(\varepsilon)$ of compounds $\mathbf{5 a - m}$ and $\mathbf{9}$ are presented in Table 2. The $\lambda_{\text {abs }}{ }^{\max }$ of compounds 5a-m and 9 was in the range of 304 321 nm. When R was H (5b, entry 2), $\lambda_{\text {abs }}{ }_{\max }$

Table 2. The spectral properties of compounds 5a-m and $\mathbf{9}$

\begin{tabular}{cccc}
\hline Entry & Compounds & $\boldsymbol{\lambda}_{\text {abs }}{ }^{\mathbf{m a x}}(\mathbf{n m})$ & $\boldsymbol{\varepsilon}(\mathbf{~} / \mathbf{m o l} / \mathbf{c m})$ \\
\hline 1 & $\mathbf{5 a}$ & 304 & $2.63 \times 10^{4}$ \\
2 & $\mathbf{5 b}$ & 307 & $2.44 \times 10^{4}$ \\
3 & $\mathbf{5 c}$ & 310 & $2.11 \times 10^{4}$ \\
4 & $\mathbf{5 d}$ & 310 & $2.14 \times 10^{4}$ \\
5 & $\mathbf{5 e}$ & 312 & $2.46 \times 10^{4}$ \\
6 & $\mathbf{5 f}$ & 312 & $2.52 \times 10^{4}$ \\
7 & $\mathbf{5 g}$ & 312 & $1.69 \times 10^{4}$ \\
8 & $\mathbf{5 h}$ & 314 & $2.89 \times 10^{4}$ \\
9 & $\mathbf{5 i}$ & 315 & $2.47 \times 10^{4}$ \\
10 & $\mathbf{5 j}$ & 316 & $2.26 \times 10^{4}$ \\
11 & $\mathbf{5 k}$ & 316 & $2.0 \times 10^{4}$ \\
12 & $\mathbf{5 l}$ & 316 & $3.30 \times 10^{4}$ \\
13 & $\mathbf{5 m}$ & 321 & $3.54 \times 10^{4}$ \\
14 & $\mathbf{9}$ & 314 & $3.60 \times 10^{4}$ \\
\hline
\end{tabular}

was $307 \mathrm{~nm}$. Introducing electron-donating substituted groups ( $\mathrm{R}=4$-ethyl (5e), 2,5-dimethoxy (5k), 4-methoxy (5l), 4-thiomethyl (5m), and compound 9; entry 5, 11-14) caused bathochromic 
shift of $5 \sim 14 \mathrm{~nm}$ compared with $\mathbf{5 b}$. While substituted groups $\mathrm{R}$ was electron-withdrawing groups $(\mathrm{R}=4$-fluoro $(\mathbf{5 c})$, 4-trifluoromethyl (5d), 4-chloro (5f), 4-cyano (5g), 4-acetyl (5h), 4-formyl (5i), and 4-vinyl (5j); entry 3, 4, 6-10), $\lambda_{\mathrm{abs}}{ }^{\max }$ generated bathochromic shift of 3 9 nm compared with $\mathbf{5 b}$. However when $\mathrm{R}$ was 2,3-dimethoxy (5a, entry 1), $\lambda_{\mathrm{abs}}{ }^{\max }$ caused hypsochromic shift of $3 \mathrm{~nm}$ compared with $\mathbf{5 b}$. From this it can be seen that variation of substituted groups $\mathrm{R}$ had effect on spectral properties and the largest bathochromic shift in $\lambda_{\mathrm{abs}}{ }^{\max }$ occurred when R was thiomethyl group (5m, entry 13$)$.

\section{Cyclic voltammetry}

Cyclic voltammetry (CV) can help us understand how some of molecules might behave as switches and memory elements. ${ }^{13}$ Hence the new products' electrochemical properties were tested by $\mathrm{CV}$ measurements. The $\mathrm{CV}$ was performed at room temperature on a $\mathrm{CHI}-650 \mathrm{C}$ voltametric analyzer using a glassy carbon electrode as working electrode, with an $\mathrm{Ag} / \mathrm{AgCl}$ reference electrode. The solutions were $1 \mathrm{mmol}$ in $\mathrm{CH}_{2} \mathrm{Cl}_{2}$ and $0.1 \mathrm{M}$ tetrabutylammonium perchlorate $\left(\mathrm{n}-\mathrm{Bu}_{4} \mathrm{NClO}_{4}\right)$ with a scan rate of $0.1 \mathrm{~V} / \mathrm{s}$.

$\mathrm{CV}$ test results of selected compounds $\mathbf{5 a}, \mathbf{5 b}, \mathbf{5 m}$ and $\mathbf{9}$ are listed in Figure 1. It is found that the four compounds showed the first reductions potential at $-1.1 \mathrm{~V}$ and the second reduction potential at $-1.82 \mathrm{~V}(\mathbf{5 a}),-1.63 \mathrm{~V}(\mathbf{5 b}),-1.71 \mathrm{~V}(\mathbf{5 m})$ and $-1.73 \mathrm{~V}(\mathbf{9})$. These gave the potential difference of $0.72 \mathrm{~V}(\mathbf{5 a}), 0.53 \mathrm{~V}(\mathbf{5 b}), 0.61 \mathrm{~V}(\mathbf{5 m})$ and $0.63 \mathrm{~V}(\mathbf{9})$. The first reduction peaks of four compounds appear to be reversible in this system. The CV of unsubstituted $\mathbf{5 b}$ were compared to the $\mathrm{CV}$ of substituted $\mathbf{5 a}, \mathbf{5 m}$ and $\mathbf{9}$ in the side of phenyl ring, the first reduction potential of $\mathbf{5 b}$ are approximately the same as $\mathbf{5 a}, \mathbf{5 m}$ and $\mathbf{9}$. However, the second reduction potential of $\mathbf{5 b}$ is higher than $\mathbf{5 a}, \mathbf{5 m}$ and $\mathbf{9}$.

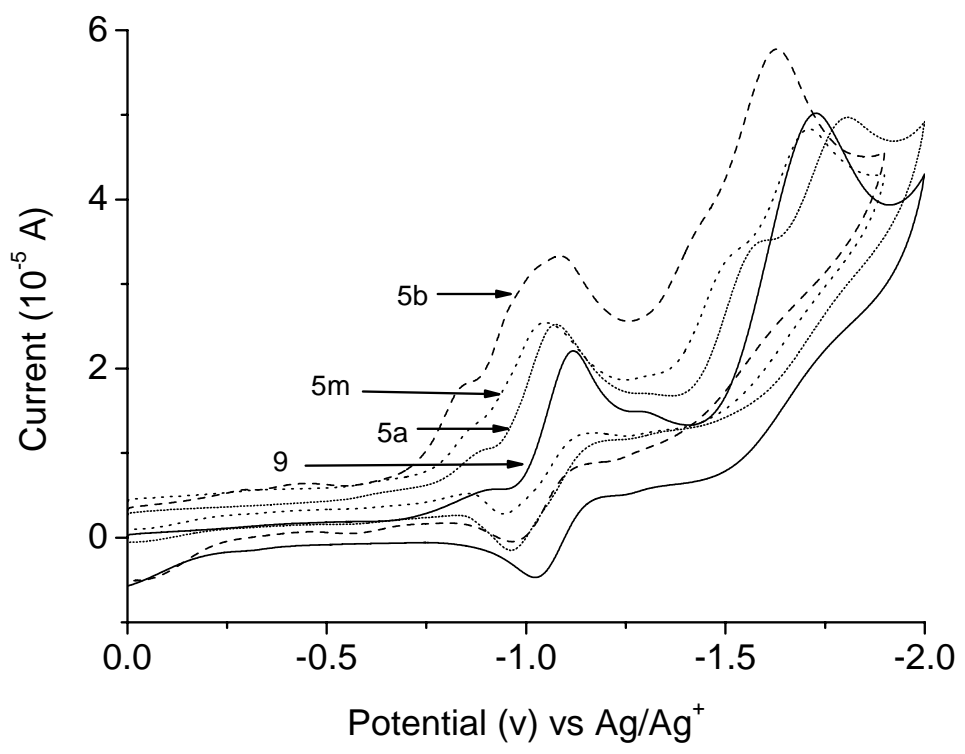

Figure 1. Cyclic voltammetry of selected compounds $\mathbf{5 a}, \mathbf{5 b}, \mathbf{5 m}$ and $\mathbf{9}$. 
The first and the second reduction potentials of these four compounds are drastically reduced compared with the reference reported "nitroaniline compound 1 "13 (the first reduction potential at $-1.7 \mathrm{~V}$ and the second reduction potential at $-2.3 \mathrm{~V}$ ). This might provide for a solid-state device with lower operating voltages than the "nitroaniline compound $\mathbf{1}$ " devices.

\section{Summary}

Fourteen novel conjugated phenylene ethynylene derivatives are designed and synthesized using convergent synthetic methodologies that are based on Sonogashira and Suzuki couplings for aryl-ethynyl and aryl-aryl bond formations. Each structure bears one nitro moiety and the "alligator clips". It was found that UV-vis maximal absorption spectra were in the range of 304 321 $\mathrm{nm}$ and compounds $\mathbf{5 m}(\mathrm{R}=\mathrm{SMe})$ have the largest.$\lambda_{\mathrm{abs}}{ }^{\max }$ than other compounds. From $\mathrm{CV}$ reduction curves we can see that selected compounds showed two obvious distinct reduction peaks. Work on further applications is currently underway in our laboratory.

\section{Experimental Section}

General. Unless stated otherwise, reactions were performed in dry, nitrogen-flushed glassware, using freshly distilled solvents. Reagent grade tetrahydrofuran (THF) were distilled from sodium benzophenone ketyl. Reagent grade chloroform $\left(\mathrm{CHCl}_{3}\right)$ and ethyl acetate (EtOAc) was used without further distillation. All other commercially available reagents were used as received. Silica gel for thin-layer chromatography was used from Qingdao Haiyang Chemical Co. Ltd. Melting points were measured by a XT5 melting point apparatus and are uncorrected. ${ }^{1} \mathrm{H}$ NMR and ${ }^{13} \mathrm{C}$ NMR spectra were recorded on a Bruker $500 \mathrm{MHz}$ spectrometer using TMS as an internal reference. Chemical shift values $(\delta)$ were given in ppm. Infrared spectra were obtained on a NEXUS670 spectrophotometer using potassium bromide pellets and are reported as wave numbers $\left(\mathrm{cm}^{-1}\right)$. High Resolution mass spectra were performed on a GCT Premier spectrometer.

\section{General procedure for the Suzuki cross coupling reaction}

Aryl halide, aryl boronic acid and tetrakis(triphenylphosphine) palladium( 0 ) (5 mol $\%$ based on aryl halide).were added in sequence to a round bottom flask equipped with a magnetic stir bar. Then the vessel was then sealed with a rubber septum, evacuated and backfilled with nitrogen $(3 \times)$. DME was added followed by aqueous $\mathrm{K}_{2} \mathrm{CO}_{3}$. The reaction heated at $85^{\circ} \mathrm{C}$ and monitored by TLC. The reaction vessel was cooled to room temperature and the mixture quenched with water and extracted with organic solvents $(3 \times)$. The combined organic layers were dried over anhydrous $\mathrm{MgSO}_{4}$ and the solvent was removed in vacuo. The crude product was then purified by flash chromatography. 


\section{General procedure for alkaline deprotection of trimethylsilyl-protected alkynes}

The TMS-protected alkyne was added to an open round bottom flask equipped with a stirring bar and tetrabutylammonium fluoride (TBAF) buffered with a mixture of acetic acid (AcOH) and acetic anhydride $\left(\mathrm{Ac}_{2} \mathrm{O}\right)$. THF was added to dissolve the organic compound. The reaction was monitored by TLC until deprotection was complete. The reaction was quenched with water and extracted with organic solvents $(3 \times)$. The combined organic layers were dried over anhydrous $\mathrm{MgSO}_{4}$ and the solvent was removed in vacuo. The crude product was then purified by flash chromatography.

\section{General procedure for the Sonogashira cross coupling reaction}

The aryl halide, tris(dibenzylideneacetone)dipalladium(0) (5 mol\% based on aryl halide) or bis(triphenylphosphine)palladium(II) dichloride (5 mol\% based on aryl halide), and copper(I) iodide (10 mol\% based on aryl halide), triphenyl phosphine (20 mol\% based on aryl halide, if necessary) were added in sequence to a round bottom flask equipped with a magnetic stir bar. Then the vessel was sealed with rubber septum, evacuated and backfilled with nitrogen $(3 \times)$. THF was added followed by Hünig's base or TEA. The terminal alkyne was then added and the reaction was heated if necessary (monitored by TLC). The reaction vessel was cooled to room temperature and the mixture quenched with water. The organic layer was diluted with organic solvent and washed with a saturated solution of $\mathrm{NH}_{4} \mathrm{Cl}(3 \times)$. The combined aqueous layers were extracted with organic solvent $(3 \times)$, dried over anhydrous $\mathrm{MgSO}_{4}$ and the solvent was removed in vacuo. The crude product was then purified by flash chromatography

4'-Ethynyl-2,3-dimethoxy-3'-nitrobiphenyl (3a). The general Suzuki cross coupling procedure was followed using 1 (0.594 g, $2 \mathrm{mmol}), 2,3$-dimethoxyphenylboronic acid (0.728 g, $4 \mathrm{mmol})$, tetrakis(triphenylphosphine) palladium(0) $(0.1156 \mathrm{~g}, 0.1 \mathrm{mmol}), \mathrm{K}_{2} \mathrm{CO}_{3}$ (aq. $\left.2 \mathrm{M} 1.05 \mathrm{~mL}\right)$, DME $(10 \mathrm{~mL})$ at $85{ }^{\circ} \mathrm{C}$ for $10 \mathrm{~h}$. Purification by flash chromatography (petroleum ether/ $\mathrm{CH}_{2} \mathrm{Cl}_{2}=15 / 1$ ) furnished ((2',3'-dimethoxy-3-nitrobiphenyl-4-yl)ethynyl)trimethylsilane (2a) as a colorless oil $(0.5321 \mathrm{~g}, 75 \%$ yield $)$. It was used directly for the next step. The general deprotection protocol was followed using 2a (0.5321 g, $1.5 \mathrm{mmol})$, TBAF $(0.5828 \mathrm{~g}, 2.25 \mathrm{mmol})$, AcOH (0.18 g, 3 $\mathrm{mmol}), \mathrm{Ac}_{2} \mathrm{O}(0.306 \mathrm{~g}, 3 \mathrm{mmol})$, THF $(10 \mathrm{~mL})$ at r.t. for $30 \mathrm{~min}$. Purification by flash chromatography (petroleum ether $\left./ \mathrm{CH}_{2} \mathrm{Cl}_{2}=8 / 1\right)$ furnished a white solid $\left(0.4059 \mathrm{~g}, 96 \%\right.$ yield). ${ }^{1} \mathrm{H}$ NMR (500 MHz, $\left.\mathrm{CDCl}_{3}\right) \delta 3.53(\mathrm{~s}, 1 \mathrm{H}, \mathrm{CH}), 3.65\left(\mathrm{~s}, 3 \mathrm{H}, \mathrm{CH}_{3}\right), 3.93\left(\mathrm{~s}, 3 \mathrm{H}, \mathrm{CH}_{3}\right), 6.96(\mathrm{q}, 1 \mathrm{H}$, $J=1.5 \mathrm{~Hz}, \operatorname{Ar}-\mathrm{H}), 6.99$ (q, $1 \mathrm{H}, J=1 \mathrm{~Hz}, \operatorname{Ar}-\mathrm{H}), 7.16$ (t, $1 \mathrm{H}, J=8 \mathrm{~Hz}, \operatorname{Ar}-\mathrm{H}), 7.72(\mathrm{~d}, 1 \mathrm{H}, J=$ $8 \mathrm{~Hz}, \mathrm{Ar}-\mathrm{H}), 7.82$ (q, $1 \mathrm{H}, J=1 \mathrm{~Hz}, \mathrm{Ar}-\mathrm{H}), 8.26$ (d, $1 \mathrm{H}, J=1 \mathrm{~Hz}, \mathrm{Ar}-\mathrm{H}) .{ }^{13} \mathrm{C}$ NMR $(125 \mathrm{MHz}$, $\left.\mathrm{CDCl}_{3}\right) \delta 56.0,60.8,78.8,85.1,113.2,115.8,121.9,124.6,125.3,132.5,133.6,135.1,139.9$, 146.6, 150.2, 153.3. Anal. Calcd for $\mathrm{C}_{16} \mathrm{H}_{13} \mathrm{NO}_{4}$ : C, 67.84; H, 4.63; N, 4.94; Found: C, 67.92; H, $4.85 ; \mathrm{N}, 5.02$.

4-Ethynyl-3-nitrobiphenyl (3b). The general Suzuki cross coupling procedure was followed using 1 (0.891 g, $3 \mathrm{mmol})$, phenylboronic acid (0.732 g, $6 \mathrm{mmol})$, tetrakis(triphenylphosphine) palladium(0) (0.1734 g, $0.15 \mathrm{mmol}), \mathrm{K}_{2} \mathrm{CO}_{3}$ (aq. $\left.2 \mathrm{M} 1.55 \mathrm{~mL}\right)$, DME $(10 \mathrm{~mL})$ at $85{ }^{\circ} \mathrm{C}$ for $2 \mathrm{~h}$. Purification by flash chromatography (petroleum ether $/ \mathrm{CH}_{2} \mathrm{Cl}_{2}=40 / 1$ ) furnished 
trimethyl((3-nitrobiphenyl-4-yl)ethynyl)silane (2b) as an orange oil (0.49 g, 55\% yield). It was used directly for the next step. The general deprotection protocol was followed using $\mathbf{2 b}(0.49 \mathrm{~g}$, $1.66 \mathrm{mmol}$ ), TBAF (0.6453 g, $2.49 \mathrm{mmol})$, AcOH (0.1992 g, $3.32 \mathrm{mmol}), \mathrm{Ac}_{2} \mathrm{O}$ (0.3386 g, 3.32 $\mathrm{mmol})$, THF $(10 \mathrm{~mL})$ at r.t. for $40 \mathrm{~min}$. Purification by flash chromatography (petroleum ether $\left./ \mathrm{CH}_{2} \mathrm{Cl}_{2}=15 / 1\right)$ furnished a pale yellow solid (0.2125 g, 57\% yield). ${ }^{1} \mathrm{H} \mathrm{NMR}(500 \mathrm{MHz}$, $\left.\mathrm{CDCl}_{3}\right) \delta 3.55(\mathrm{~s}, 1 \mathrm{H}, \mathrm{CH})$, 7.43-7.46 (m, $\left.1 \mathrm{H}, \mathrm{Ar}-\mathrm{H}\right), 7.48-7.51$ (m, $\left.2 \mathrm{H}, \mathrm{Ar}-\mathrm{H}\right)$, 7.61-7.65 (m, 2 $\mathrm{H}, \operatorname{Ar}-\mathrm{H}), 7.75$ (d, $1 \mathrm{H}, J=8.1 \mathrm{~Hz}, \operatorname{Ar}-\mathrm{H}), 7.81$ (q, $1 \mathrm{H}, J=1.8 \mathrm{~Hz}, \mathrm{Ar}-\mathrm{H}), 8.27$ (d, $1 \mathrm{H}, J=1.75$ $\mathrm{Hz}, \mathrm{Ar}-\mathrm{H}) .{ }^{13} \mathrm{C}$ NMR $\left(125 \mathrm{MHz}, \mathrm{CDCl}_{3}\right) \delta$ 78.5, 85.4, 115.8, 122.8, 127.0, 128.9, 129.2, 130.9, 135.8, 137.7, 142.7, 150.7. Anal. Calcd for $\mathrm{C}_{14} \mathrm{H}_{9} \mathrm{NO}_{2}$ : $\mathrm{C}, 75.33$; $\mathrm{H}, 4.06$; N, 6.27; Found: $\mathrm{C}$, 75.22; H, 4.19; N, 6.15.

4-Ethynyl-4'-fluoro-3-nitrobiphenyl (3c). The general Suzuki cross coupling procedure was followed using 1 (0.891 g, $3 \mathrm{mmol})$, 4-fluorophenylboronic acid (0.84 g, $6 \mathrm{mmol})$, tetrakis(triphenylphosphine) palladium(0) $(0.1272 \mathrm{~g}, 0.11 \mathrm{mmol}), \mathrm{K}_{2} \mathrm{CO}_{3}$ (aq. $2 \mathrm{M} 1.55 \mathrm{~mL}$ ), DME $(10 \mathrm{~mL})$ at $85{ }^{\circ} \mathrm{C}$ for $3 \mathrm{~h}$. Purification by flash chromatography (petroleum ether $\left./ \mathrm{CH}_{2} \mathrm{Cl}_{2}=10 / 1\right)$ furnished ((4'-fluoro-3-nitrobiphenyl-4-yl)ethynyl)trimethylsilane (2c) as colorless oil (0.4628 g, 49\% yield). It was used directly for the next step. The general deprotection protocol was followed using 2c (0.4628 g, $1.48 \mathrm{mmol})$, TBAF (0.5744 g, 2.22 $\mathrm{mmol}$ ), $\mathrm{AcOH}$ (0.1776 g, $3 \mathrm{mmol})$, $\mathrm{Ac}_{2} \mathrm{O}$ (0.3019 g, $\left.3 \mathrm{mmol}\right)$, THF (10 mL) at r.t. for $2 \mathrm{~h}$. Purification by flash chromatography (petroleum ether $/ \mathrm{CH}_{2} \mathrm{Cl}_{2}=8 / 1$ ) furnished a white solid (0.2994 g, 84\% yield). ${ }^{1} \mathrm{H}$ NMR (500 MHz, $\left.\mathrm{CDCl}_{3}\right) \delta 3.55$ (s, $\left.1 \mathrm{H}, \mathrm{CH}\right), 7.17-7.20(\mathrm{~m}, 2 \mathrm{H}$, Ar-H), 7.57-7.59 (m, $2 \mathrm{H}, \mathrm{Ar}-\mathrm{H}), 7.75$ (q, $2 \mathrm{H}, J=1.25 \mathrm{~Hz}, \mathrm{Ar}-\mathrm{H}), 8.21$ (d, $1 \mathrm{H}, J=1.0 \mathrm{~Hz}$, Ar-H). ${ }^{13} \mathrm{C}$ NMR $\left(125 \mathrm{MHz}, \mathrm{CDCl}_{3}\right) \delta 78.4,85.6,115.9,116.2,116.4(\mathrm{~d}, J=22.5 \mathrm{~Hz}), 122.7$, $128.8,128.9(\mathrm{~d}, J=8.75 \mathrm{~Hz}), 130.8,133.9,134.0(\mathrm{~d}, J=8.75 \mathrm{~Hz}), 135.9,141.7,162.3,164.3$ (d, $J=248.75 \mathrm{~Hz}$ ). Anal. Calcd for $\mathrm{C}_{14} \mathrm{H}_{8} \mathrm{FNO}_{2}: \mathrm{C}, 69.71 ; \mathrm{H}, 3.34 ; \mathrm{N}, 5.81$; Found: C, 69.82; H, $3.47 ; \mathrm{N}, 5.98$.

4-Ethynyl-3-nitro-4'-(trifluoromethyl)biphenyl (3d). The general Suzuki cross coupling procedure was followed using $1(0.891 \mathrm{~g}, 3 \mathrm{mmol})$, 4-trifluorophenylboronic acid (0.8963 g, 4.5 $\mathrm{mmol}$ ), tetrakis(triphenylphosphine) palladium(0) (0.1734 g, $0.15 \mathrm{mmol}), \mathrm{K}_{2} \mathrm{CO}_{3}$ (aq. $2 \mathrm{M} 1.55$ $\mathrm{mL})$, DME $(10 \mathrm{~mL})$ at $85{ }^{\circ} \mathrm{C}$ for $1.5 \mathrm{~h}$. Purification by flash chromatography (petroleum ether/ $\left.\mathrm{CH}_{2} \mathrm{Cl}_{2}=15 / 1\right)$ furnished trimethyl((3-nitro-4'-(trifluoromethyl)biphenyl-4-yl)ethynyl)silane (2d) as a pale yellow oil (0.628 g, 58\% yield). It was used directly for the next step. The general deprotection protocol was followed using 2d (0.3701 g, $1.02 \mathrm{mmol})$, TBAF (0.3963 g, 1.53 $\mathrm{mmol}$ ), AcOH (0.1224 g, $2.04 \mathrm{mmol}), \mathrm{Ac}_{2} \mathrm{O}$ (0.2081 g, $\left.2.04 \mathrm{mmol}\right)$, THF (10 mL) at r.t. for $2 \mathrm{~h}$. Purification by flash chromatography (petroleum ether $/ \mathrm{CH}_{2} \mathrm{Cl}_{2}=10 / 1$ ) furnished a pale yellow solid (0.2887 g, 97\% yield). ${ }^{1} \mathrm{H}$ NMR $\left(500 \mathrm{MHz}, \mathrm{CDCl}_{3}\right) \delta 3.60(\mathrm{~s}, 1 \mathrm{H}, \mathrm{CH}), 7.72-7.80(\mathrm{~m}, 4 \mathrm{H}$, Ar-H), 7.83 (q, $2 \mathrm{H}, J=1.4 \mathrm{~Hz}, \mathrm{Ar}-\mathrm{H}), 8.29$ (d, $1 \mathrm{H}, J=1.4 \mathrm{~Hz}, \mathrm{Ar}-\mathrm{H}) .{ }^{13} \mathrm{C}$ NMR $(125 \mathrm{MHz}$, $\left.\mathrm{CDCl}_{3}\right) \delta 78.3,86.2,116.9,123.1,126.2,126.2,126.3,127.5,131.1,136.1,141.1,141.3$. Anal. Calcd for $\mathrm{C}_{15} \mathrm{H}_{8} \mathrm{~F}_{3} \mathrm{NO}_{2}$ : C, 61.86; H, 2.77; N, 4.81; Found: C, 61.97; H, 2.89; N, 4.75 .

4'-Ethyl-4-ethynyl-3-nitrobiphenyl (3e). The general Suzuki cross coupling procedure was followed using 1 (0.297 $\mathrm{g}, 1 \mathrm{mmol})$, 4-ethylphenylboronic acid $(0.3 \mathrm{~g}, 2 \mathrm{mmol})$, 
tetrakis(triphenylphosphine) palladium(0) $(0.0578 \mathrm{~g}, 0.05 \mathrm{mmol}), \mathrm{K}_{2} \mathrm{CO}_{3}$ (aq. $2 \mathrm{M} 0.55 \mathrm{~mL}$ ), DME $(10 \mathrm{~mL})$ at $85{ }^{\circ} \mathrm{C}$ for $1 \mathrm{~h}$. Purification by flash chromatography (petroleum ether $\left./ \mathrm{CH}_{2} \mathrm{Cl}_{2}=10 / 1\right)$ furnished ((4'-ethyl-3-nitrobiphenyl-4-yl)ethynyl)trimethylsilane (2e) as a pale yellow oil $(0.2712 \mathrm{~g}, 84 \%$ yield). It was used directly for the next step. The general deprotection protocol was followed using 2e (0.5206 g, $1.61 \mathrm{mmol})$, TBAF (0.6216 g, 2.42 $\mathrm{mmol}$ ), $\mathrm{AcOH}$ (0.192 g, $3.22 \mathrm{mmol}), \mathrm{Ac}_{2} \mathrm{O}$ (0.3264 g, $\left.3.22 \mathrm{mmol}\right)$, THF (10 mL) at r.t. for $2 \mathrm{~h}$. Purification by flash chromatography (petroleum ether/ $\mathrm{CH}_{2} \mathrm{Cl}_{2}=10 / 1$ ) furnished a pale yellow solid (0.3144 g, 78\% yield). ${ }^{1} \mathrm{H}$ NMR (500 MHz, $\left.\mathrm{CDCl}_{3}\right) \delta 1.31\left(\mathrm{t}, 3 \mathrm{H}, J=7.6 \mathrm{~Hz}, \mathrm{CH}_{3}\right), 2.75$ (q, $\left.2 \mathrm{H}, J=7.6 \mathrm{~Hz}, \mathrm{CH}_{2}\right), 3.56(\mathrm{~s}, 1 \mathrm{H}, \mathrm{CH}), 7.35$ (d, $\left.2 \mathrm{H}, J=8.05 \mathrm{~Hz}, \mathrm{~A} \mathrm{r}-\mathrm{H}\right), 7.57$ (d, $2 \mathrm{H}, J=$ $8.1 \mathrm{~Hz}, \mathrm{Ar}-\mathrm{H}), 7.75$ (d, $1 \mathrm{H}, J=8.05 \mathrm{~Hz}, \mathrm{Ar}-\mathrm{H}), 7.82$ (q, $1 \mathrm{H}, J=1.7 \mathrm{~Hz}, \mathrm{Ar}-\mathrm{H}), 8.28$ (d, $1 \mathrm{H}, J=$ $1.65 \mathrm{~Hz}, \mathrm{Ar}-\mathrm{H}) .{ }^{13} \mathrm{C} \mathrm{NMR}\left(125 \mathrm{MHz}, \mathrm{CDCl}_{3}\right) \delta 15.4,28.5,78.6,85.2,115.5,122.6,126.9,128.8$, 130.7, 135.1, 135.8, 142.7, 145.4, 150.7. Anal. Calcd for $\mathrm{C}_{16} \mathrm{H}_{13} \mathrm{NO}_{2}$ : C, 76.48; H, 5.21; N, 5.57; Found: C, 76.34; H, 5.19; N, 5.45.

4'-Chloro-4-ethynyl-3-nitrobiphenyl (3f). The general Suzuki cross coupling procedure was followed using 1 (0.891 g, $3 \mathrm{mmol})$, 4-chlorophenylboronic acid (0.6234 g, $4 \mathrm{mmol})$, tetrakis(triphenylphosphine) palladium(0) $(0.1734 \mathrm{~g}, 0.15 \mathrm{mmol}), \mathrm{K}_{2} \mathrm{CO}_{3}$ (aq. $2 \mathrm{M} 1.55 \mathrm{~mL}$ ), DME $(10 \mathrm{~mL})$ at $85{ }^{\circ} \mathrm{C}$ for $3 \mathrm{~h}$. Purification by flash chromatography (petroleum ether $\left./ \mathrm{CH}_{2} \mathrm{Cl}_{2}=5 / 1\right)$ furnished ((4'-chloro-3-nitrobiphenyl-4-yl)ethynyl)trimethylsilane (2f) as a yellow solid $(0.2168 \mathrm{~g}, 22 \%$ yield). It was used directly for the next step. The general deprotection protocol was followed using $2 \mathrm{f}(0.2168 \mathrm{~g}, 0.66 \mathrm{mmol})$, TBAF $(0.2589 \mathrm{~g}, 1 \mathrm{mmol})$, $\mathrm{AcOH}(0.08 \mathrm{~g}, 1.3 \mathrm{mmol}), \mathrm{Ac}_{2} \mathrm{O}(0.1346 \mathrm{~g}, 1.3 \mathrm{mmol})$, THF (10 mL) at r.t. for $2 \mathrm{~h}$. Purification by flash chromatography (petroleum ether $\left./ \mathrm{CH}_{2} \mathrm{Cl}_{2}=3 / 1\right)$ furnished a yellow solid $(0.17 \mathrm{~g}, 91 \%$ yield). ${ }^{1} \mathrm{H}$ NMR (500 MHz, $\left.\mathrm{CDCl}_{3}\right) \delta 3.57$ (s, $\left.1 \mathrm{H}, \mathrm{CH}\right), 7.47$ (d, $\left.2 \mathrm{H}, J=8.5 \mathrm{~Hz}, \mathrm{Ar}-\mathrm{H}\right), 7.55$ (d, $2 \mathrm{H}, J=8.5 \mathrm{~Hz}, \mathrm{Ar}-\mathrm{H}), 7.74-7.78(\mathrm{~m}, 2 \mathrm{H}, \mathrm{Ar}-\mathrm{H}), 8.23$ (d, $1 \mathrm{H}, J=1.2 \mathrm{~Hz}, \mathrm{Ar}-\mathrm{H}) .{ }^{13} \mathrm{C}$ NMR $(125$ $\left.\mathrm{MHz}_{\mathrm{CDCl}}\right) \delta$ 78.4. 85.8, 116.2, 122.7, 128.3, 129.5, 130.8, 135.3, 136.0, 136.1, 141.4, 150.7. Anal. Calcd for $\mathrm{C}_{14} \mathrm{H}_{8} \mathrm{ClNO}_{2}$ : C, 65.26; H, 3.13; N, 5.44; Found: C, 65.20; H, 3.19; N, 5.52.

4'-Ethynyl-3'-nitrobiphenyl-4-carbonitrile (3g). The general Suzuki cross coupling procedure was followed using 1 (0.891 g, $3 \mathrm{mmol})$, 4-cyanophenylboronic acid (0.3563 g, $2.4 \mathrm{mmol})$, tetrakis(triphenylphosphine) palladium(0) $(0.1734 \mathrm{~g}, 0.15 \mathrm{mmol}), \mathrm{K}_{2} \mathrm{CO}_{3}$ (aq. $2 \mathrm{M} 1.55 \mathrm{~mL}$ ), DME $(10 \mathrm{~mL})$ at $85{ }^{\circ} \mathrm{C}$ for $2 \mathrm{~h}$. Purification by flash chromatography (petroleum ether $\left./ \mathrm{CH}_{2} \mathrm{Cl}_{2}=3 / 1\right)$ furnished $(\mathbf{2 g})$ as a pale yellow solid $(0.177 \mathrm{~g}, 23 \%$ yield based on boronic acid). It was used directly for the next step. The general deprotection protocol was followed using $2 \mathrm{~g}$ (0.177 g, $0.55 \mathrm{mmol})$, TBAF (0.2149 g, $0.83 \mathrm{mmol}), \mathrm{AcOH}(0.066 \mathrm{~g}, 1.1 \mathrm{mmol}), \mathrm{Ac}_{2} \mathrm{O}$ $(0.1122 \mathrm{~g}, 1.1 \mathrm{mmol})$, THF $(10 \mathrm{~mL})$ at r.t. for $1 \mathrm{~h}$. Purification by flash chromatography (petroleum ether/ $\left.\mathrm{CH}_{2} \mathrm{Cl}_{2}=1 / 1\right)$ furnished a yellow solid $\left(0.1267 \mathrm{~g}, 93 \%\right.$ yield). ${ }^{1} \mathrm{H}$ NMR (500 $\left.\mathrm{MHz} \mathrm{CDCl}_{3}\right) \delta 3.60$ (s, $\left.1 \mathrm{H}, \mathrm{CH}\right), 7.73$ (d, $\left.2 \mathrm{H}, J=8 \mathrm{~Hz}, \mathrm{Ar}-\mathrm{H}\right), 7.80$ (d, $\left.4 \mathrm{H}, J=9 \mathrm{~Hz}, \mathrm{Ar}-\mathrm{H}\right)$, 8.27 (s, $1 \mathrm{H}, \mathrm{Ar}-\mathrm{H}) .{ }^{13} \mathrm{C} \mathrm{NMR}\left(125 \mathrm{MHz}, \mathrm{CDCl}_{3}\right) \delta 78.1,86.6,112.9,117.4,118.2,123.1,127.8$, 130.9, 133.0, 136.2, 140.5, 142.1, 150.9. Anal. Calcd for $\mathrm{C}_{15} \mathrm{H}_{8} \mathrm{~N}_{2} \mathrm{O}_{2}$ : C, 72.58; H, 3.25; N, 11.28; Found: C, 72.34; H, 3.43; N, 11.05 .

1-(4'-Ethynyl-3'-nitrobiphenyl-4-yl)ethanone (3h). The general Suzuki cross coupling 
procedure was followed using $1(0.4638 \mathrm{~g}, 1.5 \mathrm{mmol})$, 4-acetylphenylboronic acid (0492 $\mathrm{g}, 3$ mmol), tetrakis(triphenylphosphine) palladium(0) (0.0867 g, $0.075 \mathrm{mmol}), \mathrm{K}_{2} \mathrm{CO}_{3}$ (aq. $2 \mathrm{M} 0.8$ $\mathrm{mL})$, DME $(10 \mathrm{~mL})$ at $85{ }^{\circ} \mathrm{C}$ for $2.5 \mathrm{~h}$. Purification by flash chromatography (petroleum ether $\left./ \mathrm{CH}_{2} \mathrm{Cl}_{2}=8 / 1\right)$ furnished a white solid $(0.1254 \mathrm{~g})$. The structure is characterized as $\mathbf{3 h}$ using NMR and the overall yield is $31 \%$ of the two steps. ${ }^{1} \mathrm{H} \mathrm{NMR}\left(500 \mathrm{MHz}, \mathrm{CDCl}_{3}\right) \delta 2.66(\mathrm{~s}, 3 \mathrm{H}$, $\mathrm{CH}_{3}$ ), 3.60 (s, $\left.1 \mathrm{H}, \mathrm{CH}\right), 7.72$ (d, $\left.2 \mathrm{H}, J=8.25 \mathrm{~Hz}, \mathrm{Ar}-\mathrm{H}\right), 7.79$ (d, $\left.1 \mathrm{H}, J=8.05 \mathrm{~Hz}, \mathrm{Ar}-\mathrm{H}\right), 7.85$ (q, $1 \mathrm{H}, J=1.7 \mathrm{~Hz}, \mathrm{Ar}-\mathrm{H}), 8.09$ (d, $2 \mathrm{H}, J=8.3 \mathrm{~Hz}, \mathrm{Ar}-\mathrm{H}), 8.31(\mathrm{~d}, 1 \mathrm{H}, J=1.6 \mathrm{~Hz}, \mathrm{Ar}-\mathrm{H}) .{ }^{13} \mathrm{C}$ NMR $\left(125 \mathrm{MHz}, \mathrm{CDCl}_{3}\right) \delta$ 26.7, 78.3, 86.2, 116.9, 123.1, 127.3, 129.2, 131.1, 136.1, 137.2, 141.3, 142.0, 150.8, 197.3. Anal. Calcd for $\mathrm{C}_{16} \mathrm{H}_{11} \mathrm{NO}_{3}$ : C, 72.45; H, 4.18; N, 5.28; Found: C, 72.56; H, 4.29; N, 5.09 .

4'-Ethynyl-3'-nitrobiphenyl-4-carbaldehyde (3i). The general Suzuki cross coupling procedure was followed using 1 (0.891 g, $3 \mathrm{mmol})$, 4-formylphenylboronic acid (0.937 g, $6 \mathrm{mmol})$, tetrakis(triphenylphosphine) palladium(0) $(0.1734 \mathrm{~g}, 0.15 \mathrm{mmol}), \mathrm{K}_{2} \mathrm{CO}_{3}$ (aq. $2 \mathrm{M} 1.55 \mathrm{~mL}$ ), DME $(10 \mathrm{~mL})$ at $85{ }^{\circ} \mathrm{C}$ for $2 \mathrm{~h}$. Purification by flash chromatography (petroleum ether $\left./ \mathrm{CH}_{2} \mathrm{Cl}_{2}=2 / 1\right)$ furnished 3'-nitro-4'-((trimethylsilyl)ethynyl)biphenyl-4-carbaldehyde (2i) as a pale yellow solid $(0.5537 \mathrm{~g}, 57 \%$ yield $)$. It was used directly for the next step. The general deprotection protocol was followed using $2 \mathbf{i}(0.5537 \mathrm{~g}, 1.72 \mathrm{mmol})$, TBAF (0.6681 g, 2.58 $\mathrm{mmol}$ ), AcOH (0.2064 g, $3.44 \mathrm{mmol}), \mathrm{Ac}_{2} \mathrm{O}$ (0.3509 g, $\left.3.44 \mathrm{mmol}\right)$, THF (10 mL) at r.t. for $2 \mathrm{~h}$. Purification by flash chromatography (petroleum ether $/ \mathrm{CH}_{2} \mathrm{Cl}_{2}=1 / 1$ ) furnished a yellow solid (0.3796 g, 88\% yield). ${ }^{1} \mathrm{H}$ NMR (500 MHz, $\left.\mathrm{CDCl}_{3}\right) \delta 3.61(\mathrm{~s}, 1 \mathrm{H}, \mathrm{CH}), 7.80-7.96(\mathrm{~m}, 4 \mathrm{H}$, Ar-H), 8.02 (d, $2 \mathrm{H}, J=7.5 \mathrm{~Hz}, \mathrm{Ar}-\mathrm{H}), 8.32$ (s, $1 \mathrm{H}, \mathrm{Ar}-\mathrm{H}), 10.10$ (s, $1 \mathrm{H}, \mathrm{CHO}) .{ }^{13} \mathrm{C}$ NMR $(125$ $\mathrm{MHz}_{\mathrm{CDCl}}$ ) $\delta$ 78.3, 86.4, 123.2, 127.7, 127.9, 130.5, 131.2, 136.2, 136.4, 141.1, 143.4, 191.4. Anal. Calcd for $\mathrm{C}_{15} \mathrm{H}_{9} \mathrm{NO}_{3}$ : C, 71.71; H, 3.61; N, 5.58; Found: C, 71.61; H, 3.83; N, 5.42.

4-Ethynyl-3-nitro-4'-vinylbiphenyl (3j). The general Suzuki cross coupling procedure was followed using 1 (0.594 g, $2 \mathrm{mmol})$, 4-vinylphenylboronic acid (0.444 g, $3 \mathrm{mmol})$, tetrakis(triphenylphosphine) palladium(0) (0.1156 g, $0.1 \mathrm{mmol}$ ), $\mathrm{K}_{2} \mathrm{CO}_{3}$ (aq. $2 \mathrm{M} 1.05 \mathrm{~mL}$ ), DME $(10 \mathrm{~mL})$ at $85^{\circ} \mathrm{C}$ for $4.5 \mathrm{~h}$. Purification by flash chromatography (petroleum ether/ $\mathrm{CH}_{2} \mathrm{Cl}_{2}=10 / 1$ ) furnished trimethyl((3-nitro-4'-vinylbiphenyl-4-yl)ethynyl)silane (2j) as a pale yellow oil $(0.0689$ $\mathrm{g}, 11 \%$ yield). It was used directly for the next step. The general deprotection protocol was followed using $2 \mathbf{j}$ (0.0689 g, $0.21 \mathrm{mmol})$, TBAF (0.10 g, $0.32 \mathrm{mmol})$, AcOH (0.03 g, $0.42 \mathrm{mmol})$, $\mathrm{Ac}_{2} \mathrm{O}(0.045 \mathrm{~g}, 0.42 \mathrm{mmol})$, THF $(10 \mathrm{~mL})$ at r.t. for $1 \mathrm{~h}$. Purification by flash chromatography (petroleum ether/ $\left.\mathrm{CH}_{2} \mathrm{Cl}_{2}=8 / 1\right)$ furnished a yellow solid (0.0574 g, 96\% yield). ${ }^{1} \mathrm{H}$ NMR (500 $\left.\mathrm{MHz} \mathrm{CDCl}_{3}\right) \delta 3.55(\mathrm{~s}, 1 \mathrm{H}, \mathrm{CH}), 5.35(\mathrm{~d}, 1 \mathrm{H}, J=11 \mathrm{~Hz}, \mathrm{CH}), 5.84(\mathrm{~d}, 1 \mathrm{H}, J=17 \mathrm{~Hz}, \mathrm{CH})$, 6.77 (q, $1 \mathrm{H}, J=11 \mathrm{~Hz}, \mathrm{CH}), 7.53$ (d, $2 \mathrm{H}, J=9 \mathrm{~Hz}, \mathrm{Ar}-\mathrm{H}), 7.59$ (d, $2 \mathrm{H}, J=9 \mathrm{~Hz}, \mathrm{Ar}-\mathrm{H}), 7.74$ $(\mathrm{d}, 1 \mathrm{H}, J=8 \mathrm{~Hz}, \mathrm{Ar}-\mathrm{H}), 7.81$ (q, $1 \mathrm{H}, J=1.5 \mathrm{~Hz}, \mathrm{Ar}-\mathrm{H}), 8.27$ (d, $1 \mathrm{H}, J=2 \mathrm{~Hz}, \mathrm{Ar}-\mathrm{H}) .{ }^{13} \mathrm{C} \mathrm{NMR}$ $\left(125 \mathrm{MHz}, \mathrm{CDCl}_{3}\right) \delta 78.6,85.5,115.2,115.8,122.7,127.1,127.2,130.7,135.9,135.9,136.9$, 138.4, 142.2, 150.8. Anal. Calcd for $\mathrm{C}_{16} \mathrm{H}_{11} \mathrm{NO}_{2}: \mathrm{C}$, 77.10; H, 4.45; N, 5.62; Found: C, 77.26; H, $4.69 ; \mathrm{N}, 5.49$.

4'-Ethynyl-2,5-dimethoxy-3'-nitrobiphenyl (3k). The general Suzuki cross coupling procedure was followed using 1 (0.1485 g, $0.5 \mathrm{mmol})$, 2,5-dimethoxyphenylboronic acid $(0.127 \mathrm{~g}, 0.7$ 
mmol), tetrakis(triphenylphosphine) palladium(0) (0.0289 g, $0.025 \mathrm{mmol}), \mathrm{K}_{2} \mathrm{CO}_{3}$ (aq. $2 \mathrm{M} 0.3$ $\mathrm{mL})$, DME $(10 \mathrm{~mL})$ at $85{ }^{\circ} \mathrm{C}$ for $10 \mathrm{~h}$. Purification by flash chromatography (petroleum ether $/ \mathrm{CH}_{2} \mathrm{Cl}_{2}=15 / 1$ ) furnished (2',5'-dimethoxy-3-nitrobiphenyl-4-yl)ethynyl)trimethylsilane (2k) as a pale yellow oil $(0.0836 \mathrm{~g}, 47 \%$ yield $)$. It was used directly for the next step. The general deprotection protocol was followed using $\mathbf{2 k}(0.0836 \mathrm{~g}, 0.24 \mathrm{mmol})$, TBAF (0.0915 g, 0.36 $\mathrm{mmol}), \mathrm{AcOH}(0.03 \mathrm{~g}, 0.5 \mathrm{mmol}), \mathrm{Ac}_{2} \mathrm{O}(0.05 \mathrm{~g}, 0.5 \mathrm{mmol})$, THF $(10 \mathrm{~mL})$ at r.t. for $1 \mathrm{~h}$. Purification by flash chromatography (petroleum ether $/ \mathrm{CH}_{2} \mathrm{Cl}_{2}=3 / 1$ ) furnished a yellow solid (0.0591 g, 87\% yield). ${ }^{1} \mathrm{H}$ NMR (500 MHz, $\left.\mathrm{CDCl}_{3}\right) \delta 3.52(\mathrm{~s}, 1 \mathrm{H}, \mathrm{CH}), 3.78\left(\mathrm{~s}, 3 \mathrm{H}, \mathrm{CH}_{3}\right), 3.82$ (s, $\left.3 \mathrm{H}, \mathrm{CH}_{3}\right), 6.89-6.96$ (m, $\left.3 \mathrm{H}, \mathrm{Ar}-\mathrm{H}\right), 7.70$ (d, $\left.1 \mathrm{H}, J=8.05 \mathrm{~Hz}, \mathrm{Ar}-\mathrm{H}\right), 7.76$ (q, $1 \mathrm{H}, J=1.7$ $\mathrm{Hz}, \mathrm{Ar}-\mathrm{H}), 8.25(\mathrm{~d}, 1 \mathrm{H}, J=1.55 \mathrm{~Hz}, \mathrm{Ar}-\mathrm{H}) .{ }^{13} \mathrm{C} \mathrm{NMR}\left(125 \mathrm{MHz}, \mathrm{CDCl}_{3}\right) \delta 55.9,56.2,78.8$, 84.9, 112.8, 114.8, 115.6, 116.3, 125.5, 128.0, 133.6, 135.0, 140.1, 150.1, 150.6, 153.9. Anal. Calcd for $\mathrm{C}_{16} \mathrm{H}_{13} \mathrm{NO}_{4}$ : C, 67.84; H, 4.63; N, 4.94; Found: C, 67.70; H, 4.79; N, 4.86.

4-Ethynyl-4'-methoxy-3-nitrobiphenyl (3I). The general Suzuki cross coupling procedure was followed using 1 (0.4455 g, $1.5 \mathrm{mmol})$, 4-methoxyphenylboronic acid (0.456 g, $3 \mathrm{mmol})$, tetrakis(triphenylphosphine) palladium(0) $(0.0867 \mathrm{~g}, 0.075 \mathrm{mmol}), \mathrm{K}_{2} \mathrm{CO}_{3}$ (aq. $2 \mathrm{M} 0.8 \mathrm{~mL}$ ), DME $(10 \mathrm{~mL})$ at $85{ }^{\circ} \mathrm{C}$ for $2 \mathrm{~h}$. Purification by flash chromatography (petroleum ether $\left./ \mathrm{CH}_{2} \mathrm{Cl}_{2}=10 / 1\right)$ furnished ((4'-methoxy-3-nitrobiphenyl-4-yl)ethynyl)trimethylsilane (2l) as a pale yellow oil $(0.4071 \mathrm{~g}, 84 \%$ yield). It was used directly for the next step. The general deprotection protocol was followed using 21 (0.3559 g, $1.1 \mathrm{mmol})$, TBAF (0.4274 g, $1.65 \mathrm{mmol})$, $\mathrm{AcOH}$ (0.132 g, $2.2 \mathrm{mmol}), \mathrm{Ac}_{2} \mathrm{O}(0.2244 \mathrm{~g}, 2.2 \mathrm{mmol})$, THF (10 mL) at r.t. for $1 \mathrm{~h}$. Purification by flash chromatography (petroleum ether/ $\left.\mathrm{CH}_{2} \mathrm{Cl}_{2}=10 / 1\right)$ furnished a yellow solid $(0.26 \mathrm{~g}, 93 \%$ yield). ${ }^{1} \mathrm{H}$ NMR $\left(500 \mathrm{MHz}, \mathrm{CDCl}_{3}\right) \delta 3.53(\mathrm{~s}, 1 \mathrm{H}, \mathrm{CH}), 3.87\left(\mathrm{~s}, 3 \mathrm{H}, \mathrm{CH}_{3}\right), 7.02(\mathrm{~d}, 2 \mathrm{H}, J=1.9$ $\mathrm{Hz}, \mathrm{A} \mathrm{r}-\mathrm{H}), 7.56$ (q, $2 \mathrm{H}, J=1.9 \mathrm{~Hz}, \mathrm{Ar}-\mathrm{H}), 7.71$ (d, $1 \mathrm{H}, J=8.05 \mathrm{~Hz}, \mathrm{Ar}-\mathrm{H}), 7.76$ (q, $1 \mathrm{H}, J=$ $1.8 \mathrm{~Hz}, \mathrm{Ar}-\mathrm{H}), 8.22(\mathrm{~d}, 1 \mathrm{H}, J=1.75 \mathrm{~Hz}, \mathrm{Ar}-\mathrm{H}) .{ }^{13} \mathrm{C} \mathrm{NMR}\left(125 \mathrm{MHz}, \mathrm{CDCl}_{3}\right) \delta$ 55.4, 78.7, 85.1, $114.7,115.1,122.2,128.2,130.1,130.4,135.8,142.3,150.8,160.4$. Anal. Calcd for $\mathrm{C}_{15} \mathrm{H}_{11} \mathrm{NO}_{3}$ : C, 71.14; H, 4.38; N, 5.53; Found: C, 71.22; H, 4.47; N, 5.45.

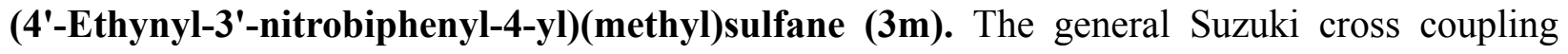
procedure was followed using $1(0.891 \mathrm{~g}, 3 \mathrm{mmol})$, 4-(methylthio)phenylboronic acid (1 g, 6 mmol), tetrakis(triphenylphosphine) palladium(0) (0.1734 g, $0.15 \mathrm{mmol}), \mathrm{K}_{2} \mathrm{CO}_{3}$ (aq. $2 \mathrm{M} 1.55$ $\mathrm{mL})$, DME $(10 \mathrm{~mL})$ at $85{ }^{\circ} \mathrm{C}$ for $23 \mathrm{~h}$. Purification by flash chromatography (petroleum ether $\left./ \mathrm{CH}_{2} \mathrm{Cl}_{2}=15 / 1\right)$ furnished trimethyl((4'-(methylthio)-3-nitrobiphenyl-4-yl)ethynyl)silane (2m) as a yellow solid $(0.17 \mathrm{~g}, 17 \%$ yield). It was used directly for the next step. The general deprotection protocol was followed using $2 \mathrm{~m}(0.17 \mathrm{~g}, 0.5 \mathrm{mmol})$, TBAF $(0.1943 \mathrm{~g}, 0.75 \mathrm{mmol})$, $\mathrm{AcOH}(0.06 \mathrm{~g}, 1 \mathrm{mmol}), \mathrm{Ac}_{2} \mathrm{O}(0.102 \mathrm{~g}, 1 \mathrm{mmol})$, THF $(10 \mathrm{~mL})$ at r.t. for $30 \mathrm{~min}$. Purification by flash chromatography (petroleum ether $\left./ \mathrm{CH}_{2} \mathrm{Cl}_{2}=3 / 1\right)$ furnished a yellow solid $(0.1122 \mathrm{~g}, 83 \%$ yield). ${ }^{1} \mathrm{H}$ NMR (500 MHz, $\left.\mathrm{CDCl}_{3}\right) \delta 2.53\left(\mathrm{~s}, 3 \mathrm{H}, \mathrm{CH}_{3}\right), 3.59(\mathrm{~s}, 1 \mathrm{H}, \mathrm{CH}), 7.35$ (d, $2 \mathrm{H}, J=$ $8.35 \mathrm{~Hz}, \mathrm{Ar}-\mathrm{H}), 7.54$ (d, $2 \mathrm{H}, J=8.35 \mathrm{~Hz}, \mathrm{Ar}-\mathrm{H}), 7.73$ (d, $1 \mathrm{H}, J=8.1 \mathrm{~Hz}, \mathrm{Ar}-\mathrm{H}), 7.78$ (q, $1 \mathrm{H}, J$ $=1.4 \mathrm{~Hz}, \mathrm{Ar}-\mathrm{H}), 8.24(\mathrm{~s}, 1 \mathrm{H}, \mathrm{Ar}-\mathrm{H}) .{ }^{13} \mathrm{C} \mathrm{NMR}\left(125 \mathrm{MHz}, \mathrm{CDCl}_{3}\right) \delta 15.4,78.6,85.4,115.6$, $122.4,126.7,127.2,130.5,134.1,135.9,140.3,142.0,150.7$. Anal. Calcd for $\mathrm{C}_{15} \mathrm{H}_{11} \mathrm{NO}_{2} \mathrm{~S}: \mathrm{C}$, 66.89; H, 4.12; N, 5.20; Found: C, 66.72; H, 4.19; N, 5.11. 
S-4-((2',3'-Dimethoxy-3-nitrobiphenyl-4-yl)ethynyl)phenyl ethanethioate (5a). The general Sonogashira cross coupling procedure was followed using 3a $(0.4059 \mathrm{~g}, 1.43 \mathrm{mmol}), \mathrm{CuI}$ (0.0272 g, $0.14 \mathrm{mmol}), \mathrm{Pd}_{2}(\mathrm{dba})_{3}(0.0655 \mathrm{~g}, 0.07 \mathrm{mmol}), \mathrm{PPh}_{3}(0.0749 \mathrm{~g}, 0.3 \mathrm{mmol}), 4(0.3975 \mathrm{~g}$, $1.43 \mathrm{mmol}),(i-P r)_{2} \mathrm{NEt}(0.7379 \mathrm{~g}, 5.7 \mathrm{mmol})$, THF $(10 \mathrm{~mL})$ at $50{ }^{\circ} \mathrm{C}$ for $24 \mathrm{~h}$. Flash chromatography (petroleum ether $\left./ \mathrm{CH}_{2} \mathrm{Cl}_{2}=3 / 1\right)$ yielded the desired adduct $(0.1668 \mathrm{~g}, 27 \%$ yield)

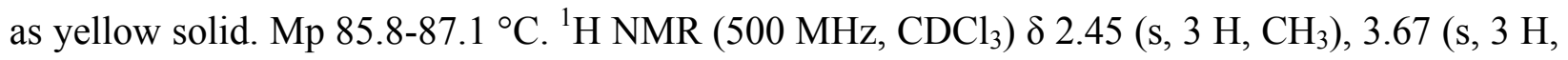
$\left.\mathrm{CH}_{3}\right), 3.94\left(\mathrm{~s}, 3 \mathrm{H}, \mathrm{CH}_{3}\right), 6.98-7.01(\mathrm{~m}, 2 \mathrm{H}, \mathrm{Ar}-\mathrm{H}), 7.17$ (t, $\left.1 \mathrm{H}, J=8 \mathrm{~Hz}, \mathrm{Ar}-\mathrm{H}\right), 7.44$ (d, $2 \mathrm{H}, J$ $=8 \mathrm{~Hz}, \mathrm{Ar}-\mathrm{H}), 7.64$ (d, $2 \mathrm{H}, J=8.5 \mathrm{~Hz}, \mathrm{Ar}-\mathrm{H}), 7.75$ (d, $1 \mathrm{H}, J=8 \mathrm{~Hz}, \mathrm{Ar}-\mathrm{H}), 7.85$ (q, $1 \mathrm{H}, J=$ $1.5 \mathrm{~Hz}, \mathrm{Ar}-\mathrm{H}), 8.32$ (d, $1 \mathrm{H}, J=1.5 \mathrm{~Hz}, \mathrm{Ar}-\mathrm{H}) .{ }^{13} \mathrm{C} \mathrm{NMR}\left(125 \mathrm{MHz}, \mathrm{CDCl}_{3}\right) \delta 30.3$ (q), 56.0 (q), 60.8 (q), 86.5 (s), 96.2 (s), 113.0 (d), 116.7 (d), 121.8 (d), 123.7 (s), 124.5 (d), 125.4 (d), 128.3 (s), 129.2 (s), 132.5 (d), 132.6 (s), 133.7 (d), 134.2 (d), 139.4 (s), 146.6 (s), 149.5 (s), 153.3 (s), 193.1 (s). FT-IR (KBr) 625 (w), $751(\mathrm{~m}), 781(\mathrm{~m}), 824(\mathrm{w}), 957(\mathrm{w}), 1001(\mathrm{~m}), 1038(\mathrm{w}), 1094$ (w), 1119 (m), 1266 (m), 1344 (m), 1385 (s), 1469 (m), 1524 (m), 1543 (m), 1621 (m), 1709 (m), $2926(\mathrm{w}) \mathrm{cm}^{-1}$. HRMS m/z: calcd for $\mathrm{C}_{24} \mathrm{H}_{19} \mathrm{NO}_{5} \mathrm{~S}$ 433.0984, found 433.0985. Anal. Calcd for $\mathrm{C}_{24} \mathrm{H}_{19} \mathrm{NO}_{5} \mathrm{~S}$ : C, 66.50; H, 4.42; N, 3.23; Found: C, 66.73; H, 4.55; N, 3.12.

S-4-((3-Nitrobiphenyl-4-yl)ethynyl)phenyl ethanethioate (5b). The general Sonogashira cross coupling procedure was followed using $3 \mathbf{b}(0.223 \mathrm{~g}, 1 \mathrm{mmol}), \mathrm{CuI}(0.019 \mathrm{~g}, 0.1 \mathrm{mmol})$, $\mathrm{Pd}_{2}(\mathrm{dba})_{3}(0.0458 \mathrm{~g}, 0.05 \mathrm{mmol}), \mathrm{PPh}_{3}(0.0524 \mathrm{~g}, 0.2 \mathrm{mmol}), 4(0.278 \mathrm{~g}, 1 \mathrm{mmol}),(\mathrm{i}-\mathrm{Pr})_{2} \mathrm{NEt}$ $(0.516 \mathrm{~g}, 4 \mathrm{mmol})$, THF $(10 \mathrm{~mL})$ at $50{ }^{\circ} \mathrm{C}$ for $17 \mathrm{~h}$. Flash chromatography (petroleum ether $\left./ \mathrm{CH}_{2} \mathrm{Cl}_{2}=3 / 1\right)$ yielded the desired adduct $(0.1978 \mathrm{~g}, 53 \%$ yield $)$ as yellow solid. Mp 133.7-134.8 ${ }^{\circ} \mathrm{C} .{ }^{1} \mathrm{H}$ NMR $\left(500 \mathrm{MHz}, \mathrm{CDCl}_{3}\right) \delta 2.45$ (s, $\left.3 \mathrm{H}, \mathrm{CH}_{3}\right), 7.41-7.47$ (m, $\left.2 \mathrm{H}, \mathrm{Ar}-\mathrm{H}\right)$, 7.50-7.54 (m, 2 H, Ar-H), 7.62-7.64 (m, 4 H, Ar-H), 7.77 (d, $1 \mathrm{H}, J=8.1 \mathrm{~Hz}, \mathrm{Ar}-\mathrm{H})$, 7.80-7.85 (m, 2 H, Ar-H), 8.33 (d, 1H, J = 1.7 Hz, Ar-H). FT-IR (KBr) $623(\mathrm{w}), 699$ (w), 761 (m), $826(\mathrm{~m})$, $951(\mathrm{w}), 1015$ (w), $1118(\mathrm{~m}), 1265$ (w), 1342 (s), 1389 (w), $1490(\mathrm{w}), 1524$ (s), 1544 (s), $1696(\mathrm{~s})$ $\mathrm{cm}^{-1}$. HRMS m/z: calcd for $\mathrm{C}_{22} \mathrm{H}_{15} \mathrm{NO}_{3} \mathrm{~S} 373.0773$, found 373.0783. Anal. Calcd for $\mathrm{C}_{22} \mathrm{H}_{15} \mathrm{NO}_{3} \mathrm{~S}$ : C, 70.76; H, 4.05; N, 3.75; Found: C, 70.67; H, 4.23; N, 3.58.

S-4-((4'-Fluoro-3-nitrobiphenyl-4-yl)ethynyl)phenyl ethanethioate $\mathbf{( 5 c ) . ~ T h e ~ g e n e r a l ~}$ Sonogashira cross coupling procedure was followed using 3c (0.2994 g, $1.24 \mathrm{mmol}), \mathrm{CuI}(0.03 \mathrm{~g}$, $0.12 \mathrm{mmol}), \mathrm{Pd}_{2}(\mathrm{dba})_{3}(0.06 \mathrm{~g}, 0.06 \mathrm{mmol}), \mathrm{PPh}_{3}(0.065 \mathrm{~g}, 0.25 \mathrm{mmol}), 4$ (0.3454 g, $\left.1.24 \mathrm{mmol}\right)$, $(i-P r)_{2} \mathrm{NEt}(0.645 \mathrm{~g}, 5 \mathrm{mmol})$, THF $(10 \mathrm{~mL})$ at $50{ }^{\circ} \mathrm{C}$ for $22 \mathrm{~h}$. Flash chromatography (petroleum ether $\left./ \mathrm{CH}_{2} \mathrm{Cl}_{2}=3 / 1\right)$ yielded the desired adduct $(0.1799 \mathrm{~g}, 37 \%$ yield $)$ as brown solid. Mp 109.7-111.2 ${ }^{\circ} \mathrm{C} .{ }^{1} \mathrm{H}$ NMR $\left(500 \mathrm{MHz}, \mathrm{CDCl}_{3}\right) \delta 2.45\left(\mathrm{~s}, 3 \mathrm{H}, \mathrm{CH}_{3}\right), 7.19(\mathrm{t}, 2 \mathrm{H}, J=8.5 \mathrm{~Hz}, \mathrm{Ar}-\mathrm{H})$, 7.44 (d, $2 \mathrm{H}, J=8 \mathrm{~Hz}, \mathrm{Ar}-\mathrm{H}), 7.60-7.64$ (m, $4 \mathrm{H}, \mathrm{Ar}-\mathrm{H}), 7.75-7.80$ (m, $2 \mathrm{H}, \mathrm{Ar}-\mathrm{H}), 8.28$ (d, $1 \mathrm{H}, J$ $=1.5 \mathrm{~Hz}, \mathrm{Ar}-\mathrm{H}) .{ }^{13} \mathrm{C} \mathrm{NMR}\left(125 \mathrm{MHz}, \mathrm{CDCl}_{3}\right) \delta 30.3(\mathrm{q}), 86.2(\mathrm{~s}), 96.7(\mathrm{~s}), 116.3$ (d, $J=21.25$ Hz), 116.9 (s), 122.9 (d), 123.5 (s), 128.8 (d, $J=8.75$ Hz), 129.3 (s), 130.9 (d), 132.5 (d), 134.0 (s), 134.2 (d), 135.1 (d), 141.1 (s), 150.0 (s), 164.3 (d, $J=247.5$ Hz), 193.1 (s). FT-IR (KBr) 620 (m), 759 (w), 826 (s), 947 (m), $1011(\mathrm{w}), 1111(\mathrm{~m}), 1163$ (m), 1232 (m), 1286 (w), 1348 (s), 1387 (w), 1517 (s), 1544 (s), 1601 (w), 1700 (s) $\mathrm{cm}^{-1}$. HRMS m/z: calcd for $\mathrm{C}_{22} \mathrm{H}_{14} \mathrm{NO}_{3} \mathrm{SF}$ 391.0678, found 391.0677. Anal. Calcd for $\mathrm{C}_{22} \mathrm{H}_{14} \mathrm{FNO}_{3} \mathrm{~S}$ : C, 67.51; H, 3.61; N, 3.58; Found: C, $67.23 ; \mathrm{H}, 4.05 ; \mathrm{N}, 3.42$. 
S-4-((3-Nitro-4'-(trifluoromethyl)biphenyl-4-yl)ethynyl)phenyl ethanethioate (5d). The general Sonogashira cross coupling procedure was followed using 3d $(0.3801 \mathrm{~g}, 1.3 \mathrm{mmol}), \mathrm{CuI}$ (0.03 g, $0.13 \mathrm{mmol}), \mathrm{Pd}_{2}(\mathrm{dba})_{3}(0.06 \mathrm{~g}, 0.065 \mathrm{mmol}), \mathrm{PPh}_{3}(0.0681 \mathrm{~g}, 0.26 \mathrm{mmol}), 4$ (0.3631 g, $1.3 \mathrm{mmol}),(i-\mathrm{Pr})_{2} \mathrm{NEt}(0.6708 \mathrm{~g}, 5.2 \mathrm{mmol})$, THF $(10 \mathrm{~mL})$ at $50{ }^{\circ} \mathrm{C}$ for $26 \mathrm{~h}$. Flash chromatography (petroleum ether $\left./ \mathrm{CH}_{2} \mathrm{Cl}_{2}=1 / 1\right)$ yielded the desired adduct $(0.351 \mathrm{~g}, 61 \%$ yield) as yellow solid. Mp 101.1-102.4 ${ }^{\circ} \mathrm{C} .{ }^{1} \mathrm{H}$ NMR $\left(500 \mathrm{MHz}, \mathrm{CDCl}_{3}\right) \delta 2.45\left(\mathrm{~s}, 3 \mathrm{H}, \mathrm{CH}_{3}\right), 7.44(\mathrm{~d}, 2$ $\mathrm{H}, J=8.15 \mathrm{~Hz}, \mathrm{Ar}-\mathrm{H}), 7.63$ (d, $2 \mathrm{H}, J=8.2 \mathrm{~Hz}, \mathrm{Ar}-\mathrm{H}), 7.74-7.78$ (m, $4 \mathrm{H}, \mathrm{Ar}-\mathrm{H}), 7.80-7.85$ (m, 2 $\mathrm{H}, \mathrm{Ar}-\mathrm{H}), 8.34$ (d, $1 \mathrm{H}, J=1.65 \mathrm{~Hz}, \mathrm{Ar}-\mathrm{H}) .{ }^{13} \mathrm{C} \mathrm{NMR}\left(125 \mathrm{MHz}, \mathrm{CDCl}_{3}\right) \delta 30.3$ (q), 86.0 (s), 97.4 (s), 117.9 (s), 123.3 (d), 123.4 (s), 126.2 (d), 127.4 (d), 129.0 (s), 129.6 (s), 131.2 (d), 132.5 (d), 134.2 (d), 135.3 (d), 140.5 (s), 141.3 (s), 150.1 (s), 193.0 (s). FT-IR (KBr) 615 (m), 830 (m), 947 (w), 1012 (w), 1074 (m), 1119 (s), 1165 (m), 1266 (w), 1325 (s), 1520 (m), 1543 (m), 1616 (w), $1706(\mathrm{~m}) \mathrm{cm}^{-1}$. HRMS m/z: calcd for $\mathrm{C}_{23} \mathrm{H}_{14} \mathrm{NO}_{3} \mathrm{SF}_{3} 441.0646$, found 441.0645. Anal. Calcd for $\mathrm{C}_{23} \mathrm{H}_{14} \mathrm{~F}_{3} \mathrm{NO}_{3} \mathrm{~S}$ : C, 62.58; H, 3.20; N, 3.17; Found: C, 62.63; H, 3.04; N, 3.32.

S-4-((4'-Ethyl-3-nitrobiphenyl-4-yl)ethynyl)phenyl ethanethioate (5e). The general Sonogashira cross coupling procedure was followed using $3 \mathbf{e}(0.1255 \mathrm{~g}, 0.5 \mathrm{mmol}), \mathrm{CuI}(0.01 \mathrm{~g}$, $0.05 \mathrm{mmol}), \mathrm{Pd}_{2}(\mathrm{dba})_{3}(0.0229 \mathrm{~g}, 0.025 \mathrm{mmol}), \mathrm{PPh}_{3}(0.0262 \mathrm{~g}, 0.1 \mathrm{mmol}), 4(0.1529 \mathrm{~g}, 0.55$ $\mathrm{mmol}),(i-\mathrm{Pr})_{2} \mathrm{NEt}(0.258 \mathrm{~g}, 2 \mathrm{mmol})$, THF $(10 \mathrm{~mL})$ at $50{ }^{\circ} \mathrm{C}$ for $7 \mathrm{~h}$. Flash chromatography (petroleum ether $\left./ \mathrm{CH}_{2} \mathrm{Cl}_{2}=3 / 1\right)$ yielded the desired adduct ( $0.09 \mathrm{~g}, 45 \%$ yield) as yellow solid. Mp 90.6-91.4 ${ }^{\circ} \mathrm{C}$. ${ }^{1} \mathrm{H}$ NMR (500 MHz, $\left.\mathrm{CDCl}_{3}\right) \delta 1.29$ (t, $\left.3 \mathrm{H}, J=7.6 \mathrm{~Hz}, \mathrm{CH}_{3}\right), 2.44\left(\mathrm{~s}, 3 \mathrm{H}, \mathrm{CH}_{3}\right)$, 2.72 (q, $2 \mathrm{H}, J=7.6 \mathrm{~Hz}, \mathrm{CH}_{2}$ ), 7.34 (t, $\left.2 \mathrm{H}, J=8.1 \mathrm{~Hz}, \mathrm{~A} \mathrm{r}-\mathrm{H}\right), 7.43$ (t, $\left.2 \mathrm{H}, J=1.65 \mathrm{~Hz}, \mathrm{Ar}-\mathrm{H}\right)$, 7.55 (t, $2 \mathrm{H}, J=8.6 \mathrm{~Hz}, \operatorname{Ar}-\mathrm{H}), 7.63(\mathrm{~d}, 2 \mathrm{H}, J=8.3 \mathrm{~Hz}, \mathrm{Ar}-\mathrm{H}), 7.75(\mathrm{~d}, 1 \mathrm{H}, J=8.1 \mathrm{~Hz}, \mathrm{Ar}-\mathrm{H})$, $7.82(\mathrm{q}, 1 \mathrm{H}, J=1.8 \mathrm{~Hz}, \mathrm{Ar}-\mathrm{H}), 8.31(\mathrm{~d}, 1 \mathrm{H}, J=1.8 \mathrm{~Hz}, \mathrm{Ar}-\mathrm{H}) .{ }^{13} \mathrm{C}$ NMR $\left(125 \mathrm{MHz}, \mathrm{CDCl}_{3}\right) \delta$ 15.4 (q), 28.6 (t), 30.3 (q), 86.5 (s), 96.4 (s), 116.5 (s), 122.8 (d), 123.7 (s), 126.9 (d), 128.8 (d), 129.2 (s), 130.8 (d), 132.5 (d), 134.2 (d), 135.0 (d), 135.2 (s), 142.1 (s), 145.4 (s), 150.0 (s), 193.1 (s). FT-IR (KBr) 616 (s), 754 (w), 825 (s), 951 (m), 1013 (w), 1114 (m), 1266 (w), 1345 (s), 1389 (m), 1519 (s), 1538 (s), 1708 (s), 2871 (w), 2928 (w), 2964 (w) cm ${ }^{-1}$. HRMS m/z: calcd for $\mathrm{C}_{24} \mathrm{H}_{19} \mathrm{NO}_{3} \mathrm{~S} 401.1086$, found 401.1082. Anal. Calcd for $\mathrm{C}_{24} \mathrm{H}_{19} \mathrm{NO}_{3} \mathrm{~S}$ : C, 71.80; H, 4.77; N, 3.49; Found: C, 71.93; H, 4.87; N, 3.29.

S-4-((4'-Chloro-3-nitrobiphenyl-4-yl)ethynyl)phenyl ethanethioate (5f). The general Sonogashira cross coupling procedure was followed using $\mathbf{3 f}(0.385 \mathrm{~g}, 1.5 \mathrm{mmol}), \mathrm{CuI}(0.03 \mathrm{~g}$, $0.15 \mathrm{mmol}), \mathrm{Pd}_{2}(\mathrm{dba})_{3}(0.0687 \mathrm{~g}, 0.075 \mathrm{mmol}), \mathrm{PPh}_{3}(0.0786 \mathrm{~g}, 0.3 \mathrm{mmol}), 4$ (0.417 g, 1.5 $\mathrm{mmol}),(\mathrm{i}-\mathrm{Pr})_{2} \mathrm{NEt}(0.774 \mathrm{~g}, 6 \mathrm{mmol})$, THF $(10 \mathrm{~mL})$ at $50{ }^{\circ} \mathrm{C}$ for $35.5 \mathrm{~h}$. Flash chromatography (petroleum ether $\left./ \mathrm{CH}_{2} \mathrm{Cl}_{2}=3 / 1\right)$ yielded the desired adduct $(0.3183 \mathrm{~g}, 52 \%$ yield) as yellow solid. Mp 115.9-117.3 ${ }^{\circ} \mathrm{C} .{ }^{1} \mathrm{H}$ NMR $\left(500 \mathrm{MHz}, \mathrm{CDCl}_{3}\right) \delta 2.45\left(\mathrm{~s}, 3 \mathrm{H}, \mathrm{CH}_{3}\right), 7.45(\mathrm{q}, 4 \mathrm{H}, \mathrm{J}=7.2 \mathrm{~Hz}$, Ar-H), 7.60 (q, $4 \mathrm{H}, J=6.5 \mathrm{~Hz}, \mathrm{Ar}-\mathrm{H}), 7.76-7.80$ (m, $2 \mathrm{H}, \mathrm{Ar}-\mathrm{H}), 8.29$ (s, $1 \mathrm{H}, \mathrm{Ar}-\mathrm{H}) .{ }^{13} \mathrm{C}$ NMR $\left(125 \mathrm{MHz}, \mathrm{CDCl}_{3}\right) \delta 30.3$ (q), 86.2 (s), 96.9 (s), 117.2 (s), 122.9 (d), 123.5 (s), 128.2 (d), 129.4 (s), 129.5 (d), 130.8 (d), 132.5 (d), 134.2 (d), 135.1 (d), 135.2 (s), 136.3 (s), 140.8 (s), 150.0 (s), 193.1 (s). FT-IR (KBr) 613 (m), $778(\mathrm{w}), 826(\mathrm{~s}), 939$ (m), 1011 (m), 1088 (s), 1279 (w), 1334 (s), 1389 (w), 1414 (w), 1487 (s), 1540 (s), 1704 (s), 2922 (w) cm $\mathrm{cm}^{-1}$ HRMS m/z: calcd for $\mathrm{C}_{22} \mathrm{H}_{14} \mathrm{NO}_{3} \mathrm{SCl} 407.0383$, found 407.0386. Anal. Calcd for $\mathrm{C}_{22} \mathrm{H}_{14} \mathrm{ClNO}_{3} \mathrm{~S}: \mathrm{C}, 64.78 ; \mathrm{H}, 3.46 ; \mathrm{N}$, 
3.43; Found: C, 64.59; H, 3.57; N, 3.29.

S-4-((4'-Cyano-3-nitrobiphenyl-4-yl)ethynyl)phenyl ethanethioate $\mathbf{( 5 g ) .}$ The general Sonogashira cross coupling procedure was followed using $3 \mathrm{~g}(0.2069 \mathrm{~g}, 0.83 \mathrm{mmol}), \mathrm{CuI}$ (0.0158 g, $0.1 \mathrm{mmol}), \mathrm{Pd}_{2}(\mathrm{dba})_{3}(0.038 \mathrm{~g}, 0.4 \mathrm{mmol}), \mathrm{PPh}_{3}(0.0435 \mathrm{~g}, 0.2 \mathrm{mmol}), 4$ (0.2307 g, $0.83 \mathrm{mmol}),(\mathrm{i}-\mathrm{Pr})_{2} \mathrm{NEt}(0.4283 \mathrm{~g}, 3.32 \mathrm{mmol})$, THF $(10 \mathrm{~mL})$ at $50{ }^{\circ} \mathrm{C}$ for $48 \mathrm{~h}$. Flash chromatography $\left(\mathrm{CH}_{2} \mathrm{Cl}_{2}\right)$ yielded the desired adduct $(0.0849 \mathrm{~g}, 26 \%$ yield $)$ as yellow solid. Mp 153.1-154.2 ${ }^{\circ} \mathrm{C} .{ }^{1} \mathrm{H}$ NMR (500 MHz, $\left.\mathrm{CDCl}_{3}\right) \delta 2.45$ (s, $\left.3 \mathrm{H}, \mathrm{CH}_{3}\right), 7.44(\mathrm{~d}, 2 \mathrm{H}, \mathrm{J}=8 \mathrm{~Hz}, \mathrm{Ar}-\mathrm{H})$, $7.63(\mathrm{~d}, 2 \mathrm{H}, J=8 \mathrm{~Hz}, \mathrm{Ar}-\mathrm{H}), 7.75$ (d, $2 \mathrm{H}, J=8 \mathrm{~Hz}, \mathrm{Ar}-\mathrm{H}), 7.81$ (q, $4 \mathrm{H}, J=6.5 \mathrm{~Hz}, \mathrm{Ar}-\mathrm{H}), 8.33$ (s, $1 \mathrm{H}, \mathrm{Ar}-\mathrm{H}) .{ }^{13} \mathrm{C} \mathrm{NMR}\left(125 \mathrm{MHz}, \mathrm{CDCl}_{3}\right) \delta 30.3$ (q), 85.9 (s), 97.8 (s), 112.7 (s), 118.3 (s), 118.4 (s), 123.3 (s), 123.3 (d), 127.7 (d), 131.1 (d), 132.6 (d), 133.0 (d), 134.3 (d), 135.4 (d), 137.4 (s), 139.8 (s), 142.2 (s), 150.1 (s), 193.0 (s). FT-IR (KBr) 623 (m), 757 (w), 825 (s), 957 (w), $1012(\mathrm{w}), 1119(\mathrm{~m}), 1253(\mathrm{w}), 1286(\mathrm{w}), 1343$ (s), 1388 (m), 1531 (s), 1607 (w), 1697 (s), $2224(\mathrm{~m}, \mathrm{CN}), 2922(\mathrm{w}), 3089(\mathrm{w}) \mathrm{cm}^{-1}$. MS (EI) m/z: 398. Anal. Calcd for $\mathrm{C}_{23} \mathrm{H}_{14} \mathrm{~N}_{2} \mathrm{O}_{3} \mathrm{~S}: \mathrm{C}$, 69.33; H, 3.54; N, 7.03; Found: C, 69.25; H, 3.59; N, 6.89.

S-4-((4'-Acetyl-3-nitrobiphenyl-4-yl)ethynyl)phenyl ethanethioate $(5 \mathrm{~h})$. The general Sonogashira cross coupling procedure was followed using $3 \mathbf{h}(0.1621 \mathrm{~g}, 0.61 \mathrm{mmol}), \mathrm{CuI}$ (0.0116 g, $0.06 \mathrm{mmol}), \mathrm{Pd}_{2}(\mathrm{dba})_{3}(0.0279 \mathrm{~g}, 0.03 \mathrm{mmol}), \mathrm{PPh}_{3}(0.0387 \mathrm{~g}, 0.12 \mathrm{mmol}), 4(0.17 \mathrm{~g}$, $0.61 \mathrm{mmol}),(i-\operatorname{Pr})_{2} \mathrm{NEt}(0.3148 \mathrm{~g}, 2.5 \mathrm{mmol})$, THF $(10 \mathrm{~mL})$ at $50{ }^{\circ} \mathrm{C}$ for $19 \mathrm{~h}$. Flash chromatography $\left(\mathrm{CH}_{2} \mathrm{Cl}_{2}\right)$ yielded the desired adduct $(0.0548 \mathrm{~g}, 22 \%$ yield $)$ as yellow solid. Mp 90.1-91.2 ${ }^{\circ} \mathrm{C} .{ }^{1} \mathrm{H}$ NMR $\left(500 \mathrm{MHz}, \mathrm{CDCl}_{3}\right) \delta 2.45\left(\mathrm{~s}, 3 \mathrm{H}, \mathrm{CH}_{3}\right), 2.66\left(\mathrm{~s}, 3 \mathrm{H}, \mathrm{CH}_{3}\right), 7.44(\mathrm{~d}, 2 \mathrm{H}$, $J=8 \mathrm{~Hz}, \mathrm{Ar}-\mathrm{H}), 7.63(\mathrm{~d}, 2 \mathrm{H}, J=8 \mathrm{~Hz}, \mathrm{Ar}-\mathrm{H}), 7.74(\mathrm{~d}, 2 \mathrm{H}, J=8.5 \mathrm{~Hz}, \mathrm{Ar}-\mathrm{H}), 7.80(\mathrm{~d}, 1 \mathrm{H}, J=$ $8 \mathrm{~Hz}, \mathrm{Ar}-\mathrm{H}), 7.87$ (d, $1 \mathrm{H}, J=8 \mathrm{~Hz}, \mathrm{Ar}-\mathrm{H}), 8.08$ (d, $2 \mathrm{H}, J=8 \mathrm{~Hz}, \mathrm{Ar}-\mathrm{H}), 8.36$ (s, $1 \mathrm{H}, \mathrm{Ar}-\mathrm{H}) .{ }^{13} \mathrm{C}$ NMR (125 MHz, $\left.\mathrm{CDCl}_{3}\right) \delta 26.3$ (q), 30.3 (q), 86.1 (s), 97.3 (s), 117.8 (s), 123.3 (d), 123.4 (s), 127.2 (d), 129.2 (d), 129.5 (s), 131.1 (d), 132.5 (d), 134.2 (d), 135.2 (d), 137.1 (s), 140.6 (s), 142.1 (s), 150.0 (s), 193.0 (s), 197.3 (s). FT-IR (KBr) 591 (m), 823 (s), 952 (m), 1011 (w), 1082 (m), $1116(\mathrm{~m}), 1188(\mathrm{w}), 1266(\mathrm{~s}), 1349$ (s), $1390(\mathrm{~m}), 1493$ (m), $1526(\mathrm{~s}), 1600$ (m), 1680 (s), 1709 (m), $2962(\mathrm{w}) \mathrm{cm}^{-1}$. Anal. Calcd for $\mathrm{C}_{24} \mathrm{H}_{17} \mathrm{NO}_{4} \mathrm{~S}$ : C, 69.38; H, 4.12; N, 3.37; Found: C, 69.24; H, 4.26; N, 3.29.

S-4-((4'-Formyl-3-nitrobiphenyl-4-yl)ethynyl)phenyl ethanethioate (5i). The general Sonogashira cross coupling procedure was followed using $3 \mathbf{i}(0.125 \mathrm{~g}, 0.5 \mathrm{mmol}), \mathrm{CuI}(0.01 \mathrm{~g}$, $0.05 \mathrm{mmol}), \mathrm{Pd}_{2}(\mathrm{dba})_{3}(0.0229 \mathrm{~g}, 0.025 \mathrm{mmol}), \mathrm{PPh}_{3}(0.0262 \mathrm{~g}, 0.1 \mathrm{mmol}), 4(0.139 \mathrm{~g}, 0.5$ $\mathrm{mmol}),(i-\mathrm{Pr})_{2} \mathrm{NEt}(0.258 \mathrm{~g}, 2 \mathrm{mmol})$, THF $(10 \mathrm{~mL})$ at $50{ }^{\circ} \mathrm{C}$ for $12 \mathrm{~h}$. Flash chromatography $\left(\mathrm{CH}_{2} \mathrm{Cl}_{2}\right)$ yielded the desired adduct $(0.0769 \mathrm{~g}, 38 \%$ yield $)$ as yellow solid. Mp 146.1-147.2 ${ }^{\circ} \mathrm{C}$. ${ }^{1} \mathrm{H}$ NMR $\left(500 \mathrm{MHz}, \mathrm{CDCl}_{3}\right) \delta 2.45\left(\mathrm{~s}, 3 \mathrm{H}, \mathrm{CH}_{3}\right), 7.44(\mathrm{~d}, 2 \mathrm{H}, J=8 \mathrm{~Hz}, \mathrm{Ar}-\mathrm{H}), 7.63(\mathrm{~d}, 2 \mathrm{H}, J=$ $8.5 \mathrm{~Hz}, \mathrm{Ar}-\mathrm{H}), 7.81$ (q, $3 \mathrm{H}, J=3 \mathrm{~Hz}, \mathrm{Ar}-\mathrm{H}), 7.88$ (q, $1 \mathrm{H}, J=2 \mathrm{~Hz}, \mathrm{Ar}-\mathrm{H}), 8.01$ (d, $2 \mathrm{H}, J=8.5$ $\mathrm{Hz}, \mathrm{Ar}-\mathrm{H}), 8.37$ (d, $1 \mathrm{H}, J=1,5 \mathrm{~Hz}, \mathrm{Ar}-\mathrm{H}), 10.09$ (s, $1 \mathrm{H}, \mathrm{Ar}-\mathrm{H}) .{ }^{13} \mathrm{C} \mathrm{NMR}\left(125 \mathrm{MHz}, \mathrm{CDCl}_{3}\right) \delta$ 30.3 (q), 86.1 (s), 97.6 (s), 118.1 (s), 123.4 (s), 123.4 (d), 127.7 (d), 129.6 (s), 130.5 (d), 131.2 (d), 132.6 (d), 134.3 (d), 135.3 (d), 136.3 (s), 140.5 (s), 143.5 (s), 150.1 (s), 191.5 (d), 193.0 (s). FT-IR (KBr) 619 (m), 763 (m), 821 (s), 956 (w), 1014 (w), 1122 (m), 1176 (m), 1216 (m), 1349 (m), 1389 (w), 1528 (m), 1604 (m), 1698 (s), 2725 (w), 2922 (w), 3087 (w) cm ${ }^{-1}$. HRMS m/z: 
calcd for $\mathrm{C}_{23} \mathrm{H}_{15} \mathrm{NO}_{4} \mathrm{~S}$ 401.0722, found 401.0715. Anal. Calcd for $\mathrm{C}_{23} \mathrm{H}_{15} \mathrm{NO}_{4} \mathrm{~S}$ : C, 68.81; $\mathrm{H}$, 3.77; N, 3.49; Found: C, 68.69; H, 3.54; N, 3.36.

S-4-((3-Nitro-4'-vinylbiphenyl-4-yl)ethynyl)phenyl ethanethioate $\mathbf{( 5 j})$. The general Sonogashira cross coupling procedure was followed using $\mathbf{3 j}(0.1312 \mathrm{~g}, 0.53 \mathrm{mmol}), \mathrm{CuI}(0.01 \mathrm{~g}$, $0.05 \mathrm{mmol}), \mathrm{Pd}_{2}(\mathrm{dba})_{3}(0.0243 \mathrm{~g}, 0.025 \mathrm{mmol}), \mathrm{PPh}_{3}(0.0278 \mathrm{~g}, 0.16 \mathrm{mmol}), 4$ (0.1473 g, 0.53 $\mathrm{mmol}),(\mathrm{i}-\mathrm{Pr})_{2} \mathrm{NEt}(0.2735 \mathrm{~g}, 2.1 \mathrm{mmol})$, THF $(10 \mathrm{~mL})$ at $50{ }^{\circ} \mathrm{C}$ for $24 \mathrm{~h}$. Flash chromatography (petroleum ether $\left./ \mathrm{CH}_{2} \mathrm{Cl}_{2}=3 / 1\right)$ yielded the desired adduct $(0.0259 \mathrm{~g}, 12 \%$ yield) as yellow solid. Mp 82.2-82.9 ${ }^{\circ} \mathrm{C} .{ }^{1} \mathrm{H}$ NMR $\left(500 \mathrm{MHz}, \mathrm{CDCl}_{3}\right) \delta 2.45\left(\mathrm{~s}, 3 \mathrm{H}, \mathrm{CH}_{3}\right), 5.35(\mathrm{~d}, 1 \mathrm{H}, J=11 \mathrm{~Hz}, \mathrm{CH})$, $5.85(\mathrm{~d}, 1 \mathrm{H}, J=17.5 \mathrm{~Hz}, \mathrm{CH}), 6.78(\mathrm{q}, 1 \mathrm{H}, J=10.75 \mathrm{~Hz}, \mathrm{CH}), 7.44$ (d, $2 \mathrm{H}, J=8 \mathrm{~Hz}, \mathrm{Ar}-\mathrm{H})$, 7.54 (d, $2 \mathrm{H}, J=8 \mathrm{~Hz}, \mathrm{Ar}-\mathrm{H}), 7.63$ (t, $4 \mathrm{H}, J=8.5 \mathrm{~Hz}, \mathrm{Ar}-\mathrm{H}), 7.77$ (d, $1 \mathrm{H}, J=8 \mathrm{~Hz}, \mathrm{Ar}-\mathrm{H}), 7.85$ (q, $1 \mathrm{H}, J=1.5 \mathrm{~Hz}, \mathrm{Ar}-\mathrm{H}), 8.34$ (d, $1 \mathrm{H}, J=1.5 \mathrm{~Hz}$, Ar-H). FT-IR (KBr) 613 (m), 693 (w), 750 (w), 825 (s), 953 (w), $1018(\mathrm{~m}), 1089$ (m), 1261 (m), 1346 (m), 1390 (w), $1536(\mathrm{~m}), 1621(\mathrm{w})$, 1652 (w), 1708 (s), 2921 (w), 2962 (w) $\mathrm{cm}^{-1}$. HRMS m/z: calcd for $\mathrm{C}_{24} \mathrm{H}_{17} \mathrm{NO}_{3} \mathrm{~S} 399.0929$, found 399.0922. Anal. Calcd for $\mathrm{C}_{24} \mathrm{H}_{17} \mathrm{NO}_{3} \mathrm{~S}$ : C, 72.16; H, 4.29; N, 3.51; Found: C, 72.28; H, $4.36 ; \mathrm{N}, 3.43$.

S-4-((2',5'-Dimethoxy-3-nitrobiphenyl-4-yl)ethynyl)phenyl ethanethioate (5k). The general Sonogashira cross coupling procedure was followed using $3 \mathbf{k}(0.0591 \mathrm{~g}, 0.21 \mathrm{mmol}), \mathrm{CuI}(0.004$ g, $0.02 \mathrm{mmol}), \mathrm{Pd}_{2}(\mathrm{dba})_{3}(0.01 \mathrm{~g}, 0.01 \mathrm{mmol}), \mathrm{PPh}_{3}(0.011 \mathrm{~g}, 0.04 \mathrm{mmol}), 4$ (0.0584 g, 0.21 $\mathrm{mmol}),(i-\mathrm{Pr})_{2} \mathrm{NEt}(0.1084 \mathrm{~g}, 0.84 \mathrm{mmol})$, THF $(10 \mathrm{~mL})$ at $50{ }^{\circ} \mathrm{C}$ for $48 \mathrm{~h}$. Flash chromatography (petroleum ether $/ \mathrm{CH}_{2} \mathrm{Cl}_{2}=1 / 1$ ) yielded the desired adduct ( $0.02 \mathrm{~g}, 22 \%$ yield) as yellow semisolid. ${ }^{1} \mathrm{H}$ NMR (500 MHz, $\left.\mathrm{CDCl}_{3}\right) \delta 2.45\left(\mathrm{~s}, 3 \mathrm{H}, \mathrm{CH}_{3}\right), 3.79\left(\mathrm{~s}, 3 \mathrm{H}, \mathrm{CH}_{3}\right), 3.82\left(\mathrm{~s}, 3 \mathrm{H}, \mathrm{CH}_{3}\right)$, 6.92-6.97 (m, 3 H, Ar-H), 7.41-7.44 (m, 2 H, Ar-H), 7.62-7.64 (m, 2 H, Ar-H), 7.70-7.73 (m, 1 H, Ar-H), 7.76-7.80 (m, $1 \mathrm{H}, \mathrm{Ar}-\mathrm{H}), 8.30$ (q, $1 \mathrm{H}, J=1.5 \mathrm{~Hz}, \operatorname{Ar}-\mathrm{H})$. FT-IR (KBr) 695 (w), 763 (w), 830 (m), 1020 (m), 1179 (m), 1214 (m), 1262 (m), 1341 (s), 1386 (m), 1492 (s), 1524 (s), 1585 (w), $1617(\mathrm{~m}), 1649(\mathrm{w}), 1707(\mathrm{~m}), 2835(\mathrm{w}), 2925(\mathrm{~m}) \mathrm{cm}^{-1}$. HRMS m/z: calcd for $\mathrm{C}_{24} \mathrm{H}_{19} \mathrm{NO}_{5} \mathrm{~S}$ 433.0984, found 433.0989.

S-4-((4'-Methoxy-3-nitrobiphenyl-4-yl)ethynyl)phenyl ethanethioate (5l). The general Sonogashira cross coupling procedure was followed using 31 (0.30 g, $1.2 \mathrm{mmol})$, CuI (0.0224 g, $0.12 \mathrm{mmol}), \mathrm{Pd}_{2}(\mathrm{dba})_{3}(0.0596 \mathrm{~g}, 0.06 \mathrm{mmol}), \mathrm{PPh}_{3}(0.0654 \mathrm{~g}, 0.36 \mathrm{mmol}), 4$ (0.3365 g, 1.2 $\mathrm{mmol}),(\mathrm{i}-\mathrm{Pr})_{2} \mathrm{NEt}(0.6601 \mathrm{~g}, 4.7 \mathrm{mmol})$, THF $(10 \mathrm{~mL})$ at $50{ }^{\circ} \mathrm{C}$ for $16 \mathrm{~h}$. Flash chromatography (petroleum ether $\left./ \mathrm{CH}_{2} \mathrm{Cl}_{2}=1 / 1\right)$ yielded the desired adduct $(0.2072 \mathrm{~g}, 44 \%$ yield) as yellow solid. Mp 80.5-81.6 ${ }^{\circ} \mathrm{C} .{ }^{1} \mathrm{H}$ NMR (500 MHz, $\left.\mathrm{CDCl}_{3}\right) \delta 2.45\left(\mathrm{~s}, 3 \mathrm{H}, \mathrm{CH}_{3}\right), 3.87\left(\mathrm{~s}, 3 \mathrm{H}, \mathrm{CH}_{3}\right), 7.02(\mathrm{~d}, 2$ $\mathrm{H}, J=8.5 \mathrm{~Hz}, \mathrm{Ar}-\mathrm{H}), 7.43$ (q, $2 \mathrm{H}, J=7.5 \mathrm{~Hz}, \mathrm{Ar}-\mathrm{H}), 7.60$ (q, $4 \mathrm{H}, J=8.25 \mathrm{~Hz}, \mathrm{Ar}-\mathrm{H}), 7.76$ (q, 2 $\mathrm{H}, J=8 \mathrm{~Hz}, \mathrm{Ar}-\mathrm{H}), 8.28$ (s, $1 \mathrm{H}, \mathrm{Ar}-\mathrm{H}) .{ }^{13} \mathrm{C} \mathrm{NMR}\left(125 \mathrm{MHz}, \mathrm{CDCl}_{3}\right) \delta 30.3$ (q), 55.4 (q), 86.5 (s), 96.3 (s), 114.7 (d), 116.0 (s), 122.4 (d), 123.7 (s), 128.2 (d), 129.2 (s), 130.2 (s), 130.4 (d), 132.5 (d), 134.2 (d), 134.9 (d), 141.8 (s), 150.0 (s), 160.4 (s), 193.1 (s). FT-IR (KBr) 616 (m), 825 (s), 949 (w), 1021 (m), 1112 (m), 1181 (m), 1253 (s), 1290 (m), 1344 (m), 1515 (s), 1542 (m), 1604 (s), 1705 (s), 2836 (w), 2926 (w), 2960 (w) cm ${ }^{-1}$. HRMS m/z: calcd for $\mathrm{C}_{23} \mathrm{H}_{17} \mathrm{NO}_{4} \mathrm{~S}$ 403.0878, found 403.0888. Anal. Calcd for $\mathrm{C}_{23} \mathrm{H}_{17} \mathrm{NO}_{4} \mathrm{~S}$ : C, 68.47; H, 4.25; N, 3.47; Found: C, $68.29 ; \mathrm{H}, 4.37 ; \mathrm{N}, 3.37$. 
S-4-((4'-(Methylthio)-3-nitrobiphenyl-4-yl)ethynyl)phenyl ethanethioate $(5 \mathrm{~m})$. The general Sonogashira cross coupling procedure was followed using $\mathbf{3 m}(0.1122 \mathrm{~g}, 0.42 \mathrm{mmol}), \mathrm{CuI}(0.008$ $\mathrm{g}, 0.04 \mathrm{mmol}), \mathrm{Pd}_{2}(\mathrm{dba})_{3}(0.0192 \mathrm{~g}, 0.02 \mathrm{mmol}), \mathrm{PPh}_{3}(0.022 \mathrm{~g}, 0.08 \mathrm{mmol}), 4(0.1168 \mathrm{~g}, 0.42$ $\mathrm{mmol}),(\mathrm{i}-\mathrm{Pr})_{2} \mathrm{NEt}(0.2167 \mathrm{~g}, 1.7 \mathrm{mmol})$, THF $(10 \mathrm{~mL})$ at $50{ }^{\circ} \mathrm{C}$ for $24 \mathrm{~h}$. Flash chromatography (petroleum ether $\left./ \mathrm{CH}_{2} \mathrm{Cl}_{2}=1 / 1\right)$ yielded the desired adduct $(0.1404 \mathrm{~g}, 79 \%$ yield) as yellow solid. Mp 88.3-89.1 ${ }^{\circ} \mathrm{C} .{ }^{1} \mathrm{H}$ NMR $\left(500 \mathrm{MHz}, \mathrm{CDCl}_{3}\right) \delta 2.45\left(\mathrm{~s}, 3 \mathrm{H}, \mathrm{CH}_{3}\right), 2.54\left(\mathrm{~s}, 3 \mathrm{H}, \mathrm{CH}_{3}\right), 7.35(\mathrm{~d}, 2$ $\mathrm{H}, J=8.05 \mathrm{~Hz}, \mathrm{Ar}-\mathrm{H}), 7.43$ (d, $2 \mathrm{H}, J=8.1 \mathrm{~Hz}, \mathrm{Ar}-\mathrm{H}), 7.56$ (d, $2 \mathrm{H}, J=8.3 \mathrm{~Hz}, \mathrm{Ar}-\mathrm{H}), 7.63$ (d, 2 $\mathrm{H}, J=8.1 \mathrm{~Hz}, \mathrm{Ar}-\mathrm{H}), 7.73-7.82(\mathrm{~m}, 2 \mathrm{H}, \mathrm{Ar}-\mathrm{H}), 8.30(\mathrm{~d}, 1 \mathrm{H}, J=1.3 \mathrm{~Hz}, \mathrm{Ar}-\mathrm{H}) .{ }^{13} \mathrm{C}$ NMR $(125$ $\mathrm{MHz}, \mathrm{CDCl}_{3}$ ) $\delta 15.5$ (q), 30.3 (q), 86.4 (s), 96.6 (s), 116.6 (s), 122.6 (d), 123.6 (s), 126.8 (d), 127.2 (d), 129.0 (s), 129.3 (s), 130.6 (d), 132.5 (d), 134.2 (d), 135.1 (d), 140.2 (s), 141.5 (s), 150.1 (s), 193.1 (s). FT-IR (KBr) 617 (m), 815 (s), 951 (w), 1011 (w), 1094 (m), 1278 (w), 1340

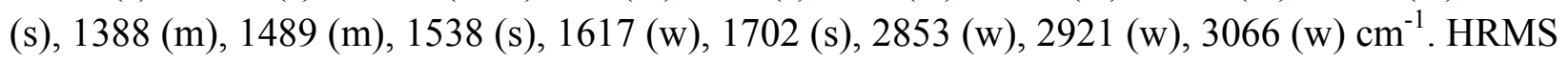
$\mathrm{m} / \mathrm{z}$ : calcd for $\mathrm{C}_{23} \mathrm{H}_{17} \mathrm{NO}_{3} \mathrm{~S}_{2} 419.0650$, found 419.0652. Anal. Calcd for $\mathrm{C}_{23} \mathrm{H}_{17} \mathrm{NO}_{3} \mathrm{~S}_{2}: \mathrm{C}, 65.85$; H, 4.08; N, 3.34; Found: C, 65.78; H, 4.19; N, 3.21.

Trimethyl((4'-(methylthio)-2-nitrobiphenyl-4-yl)ethynyl)silane (7). The general Sonogashira cross coupling procedure was followed using 6 (0.2193 g, $0.7 \mathrm{mmol}), \mathrm{CuI}(0.005 \mathrm{~g}, 0.03 \mathrm{mmol})$, $\mathrm{PdCl}_{2}\left(\mathrm{PPh}_{3}\right)_{2}(0.0238 \mathrm{~g} \mathrm{~g}, 0.03 \mathrm{mmol})$, ethynyltrimethylsilane $(0.1 \mathrm{~g}, 1 \mathrm{mmol})$, TEA $(0.2747 \mathrm{~g}$, $2.7 \mathrm{mmol})$, THF $(10 \mathrm{~mL})$ at reflux for $11 \mathrm{~h}$. Flash chromatography (petroleum ether $/ \mathrm{CH}_{2} \mathrm{Cl}_{2}=15 / 1$ ) yielded the desired adduct $(0.2315 \mathrm{~g}, 97 \%$ yield $)$ as yellow solid. ${ }^{1} \mathrm{H}$ NMR $\left(500 \mathrm{MHz}, \mathrm{CDCl}_{3}\right) \delta$ $0.29\left(\mathrm{~s}, 9 \mathrm{H}, \mathrm{CH}_{3}\right), 2.51\left(\mathrm{~s}, 3 \mathrm{H}, \mathrm{CH}_{3}\right), 7.22(\mathrm{~d}, 2 \mathrm{H}, J=8.5 \mathrm{~Hz}, \mathrm{Ar}-\mathrm{H}), 7.28(\mathrm{~d}, 2 \mathrm{H}, J=8.5 \mathrm{~Hz}$, Ar-H), 7.36 (d, $1 \mathrm{H}, J=8 \mathrm{~Hz}, \operatorname{Ar}-\mathrm{H}), 7.64$ (q, $1 \mathrm{H}, J=1.5 \mathrm{~Hz}, \operatorname{Ar}-\mathrm{H}), 7.90$ (d, $1 \mathrm{H}, J=1.5 \mathrm{~Hz}$, Ar-H). ${ }^{13} \mathrm{C}$ NMR $\left(125 \mathrm{MHz}, \mathrm{CDCl}_{3}\right) \delta 1.0,15.4,98.0,102.0,123.6,126.4,127.4,128.2,131.7$, 133.1, 135.2, 135.3, 139.7, 149.0. FT-IR (KBr) $758(\mathrm{~m}), 810$ (s), $846(\mathrm{~s}), 1022$ (m), $1096(\mathrm{~m})$, 1256 (m), 1356 (m), 1478 (m), 1541 (m), 1595 (w), 2960 (w) cm $\mathrm{cm}^{-1}$. Anal. Calcd for $\mathrm{C}_{18} \mathrm{H}_{19} \mathrm{NO}_{2} \mathrm{SSi}$ : C, 63.31; H, 5.61; N, 4.10; Found: C, 63.20; H, 5.82; N, 4.03.

(4'-Ethynyl-2'-nitrobiphenyl-4-yl)(methyl)sulfane (8). The general deprotection protocol was followed using 7 (0.2319 g, $0.7 \mathrm{mmol})$, TBAF (0.2642 g, $1.02 \mathrm{mmol})$, AcOH (0.0816 g, 1.36 $\mathrm{mmol}), \mathrm{Ac}_{2} \mathrm{O}(0.1387 \mathrm{~g}, 1.36 \mathrm{mmol})$, THF $(10 \mathrm{~mL})$ at r.t. for 1 h. Purification by flash chromatography (petroleum ether $\left./ \mathrm{CH}_{2} \mathrm{Cl}_{2}=10 / 1\right)$ furnished a pale yellow solid $(0.1471 \mathrm{~g}, 80 \%$ yield). ${ }^{1} \mathrm{H}$ NMR $\left(500 \mathrm{MHz}, \mathrm{CDCl}_{3}\right) \delta 2.51\left(\mathrm{~s}, 3 \mathrm{H}, \mathrm{CH}_{3}\right), 3.23(\mathrm{~s}, 1 \mathrm{H}, \mathrm{CH}), 7.22(\mathrm{~d}, 2 \mathrm{H}, J=$ $8.35 \mathrm{~Hz}, \mathrm{Ar}-\mathrm{H}), 7.29$ (d, $2 \mathrm{H}, J=8.3 \mathrm{~Hz}, \mathrm{Ar}-\mathrm{H}), 7.39$ (d, $1 \mathrm{H}, J=7.95 \mathrm{~Hz}, \mathrm{Ar}-\mathrm{H}), 7.68$ (q, $1 \mathrm{H}, J$ $=1.4 \mathrm{~Hz}, \operatorname{Ar}-\mathrm{H}), 7.93(\mathrm{~d}, 1 \mathrm{H}, J=1.1 \mathrm{~Hz}, \mathrm{Ar}-\mathrm{H})$. FT-IR (KBr) 815 (w), 1020 (w), 1099 (w), $1170(\mathrm{~m}), 1262(\mathrm{w}), 1353(\mathrm{~m}), 1405(\mathrm{~m}), 1568(\mathrm{~s}), 1663(\mathrm{~m}) \mathrm{cm}^{-1}$. Anal. Calcd for $\mathrm{C}_{15} \mathrm{H}_{11} \mathrm{NO}_{2} \mathrm{~S}$ : C, 66.89; H, 4.12; N, 5.20; Found: C, 66.77; H, 4.24; N, 5.24.

S-4-((4'-(Methylthio)-2-nitrobiphenyl-4-yl)ethynyl)phenyl ethanethioate (9). The general Sonogashira cross coupling procedure was followed using 8 (0.1471 g, $0.55 \mathrm{mmol}), \mathrm{CuI}(0.0105$ $\mathrm{g}, 0.06 \mathrm{mmol}), \mathrm{Pd}_{2}(\mathrm{dba})_{3}(0.0252 \mathrm{~g}, 0.03 \mathrm{mmol}), \mathrm{PPh}_{3}(0.03 \mathrm{~g}, 0.11 \mathrm{mmol}), 4(0.152 \mathrm{~g}, 0.55$ $\mathrm{mmol}),(\mathrm{i}-\mathrm{Pr})_{2} \mathrm{NEt}(0.2838 \mathrm{~g}, 2.2 \mathrm{mmol})$, THF $(10 \mathrm{~mL})$ at $50{ }^{\circ} \mathrm{C}$ for $48 \mathrm{~h}$. Flash chromatography (petroleum ether $\left./ \mathrm{CH}_{2} \mathrm{Cl}_{2}=3 / 1\right)$ yielded the desired adduct $(0.1044 \mathrm{~g}, 45 \%$ yield) as yellow solid. Mp 77.4-77.9 ${ }^{\circ} \mathrm{C} .{ }^{1} \mathrm{H}$ NMR $\left(500 \mathrm{MHz}, \mathrm{CDCl}_{3}\right) \delta 2.45\left(\mathrm{~s}, 3 \mathrm{H}, \mathrm{CH}_{3}\right), 2.52\left(\mathrm{~s}, 3 \mathrm{H}, \mathrm{CH}_{3}\right)$, 7.24-7.26 
(m, $2 \mathrm{H}, \mathrm{Ar}-\mathrm{H}), 7.30(\mathrm{~d}, 2 \mathrm{H}, J=8.35 \mathrm{~Hz}, \mathrm{Ar}-\mathrm{H}), 7.41-7.44(\mathrm{~m}, 3 \mathrm{H}, \mathrm{Ar}-\mathrm{H}), 7.58(\mathrm{~d}, 2 \mathrm{H}, J=$ $8.25 \mathrm{~Hz}, \mathrm{Ar}-\mathrm{H}), 7.72$ (q, $1 \mathrm{H}, J=1.6 \mathrm{~Hz}, \mathrm{Ar}-\mathrm{H}), 7.98$ (d, $1 \mathrm{H}, J=1.45 \mathrm{~Hz}, \mathrm{Ar}-\mathrm{H}) .{ }^{13} \mathrm{C}$ NMR $(125$ $\mathrm{MHz}, \mathrm{CDCl}_{3}$ ) $\delta 15.4$ (q), 30.3 (q), 88.3 (s), 91.4 (s), 123.3 (s), 123.4 (s), 126.4 (d), 127.0 (d), 128.2 (d), 128.9 (s), 131.9 (d), 132.3 (d), 133.0 (s), 134.3 (d), 134.9 (d), 135.4 (s), 139.7 (s), 149.1 (s), 193.1 (s). FT-IR (KBr) 822 (s), 952 (m), 1095 (m), 1188 (w), 1262 (w), 1355 (m), 1431 (w), 1486 (m), 1534 (s), 1593 (w), 1709 (s), 2855 (w), 2921 (w), 2961 (w) cm ${ }^{-1}$. HRMS $\mathrm{m} / \mathrm{z}$ : calcd for $\mathrm{C}_{23} \mathrm{H}_{17} \mathrm{NO}_{3} \mathrm{~S}_{2} 419.0650$, found 419.0650. Anal. Calcd for $\mathrm{C}_{23} \mathrm{H}_{17} \mathrm{NO}_{3} \mathrm{~S}_{2}$ : C, 65.85; H, 4.08; N, 3.34; Found: C, 65.72; H, 4.21; N, 3.24.

\section{Acknowledgements}

We gratefully acknowledge the National Natural Science Foundation of China (20873041) and Shanghai Leading Academic Discipline Project (B409) for financial support.

\section{References}

1. (a) Chen, X. D.; Jeon, Y. M.; Jang, J. W.; Qin, L. D. Huo, F. W.; Wei, W.; Mirkin, C. A. J. Am. Chem. Soc. 2008, 130, 8166. (b) Lewis, P. A.; Inman, C. E.; Maya, F.; Tour, J. M.; Hutchison, J. E.; Weiss, P. S. J. Am. Chem. Soc. 2005, 127, 17421. (c) Lewis, P. A.; Inman, C. E.; Yao, Y.; Tour, J. M.; Hutchison, J. E.; Weiss, P. S. J. Am. Chem. Soc. 2004, 126, 12214. (d) Joachim, C.; Gimzewski, J. K.; Aviram, A. Nature 2000, 408, 541. (e) Tour, J. M. Acc. Chem. Res. 2000, 33, 791.

2. (a) Fan, F. F.; Yang, J.; Dirk, S. M.; Price, D. W.; Kosynkin, D.; Tour, J. M.; Bard, A. J. J. Am. Chem. Soc. 2001, 123 (10), 2454. (b) Fan, F. F.; Yang, J.; Cai, L.; Price, D. W.; S Dirk, H. M.; Kosynkin, D. V.; Yao, Y.; Rawlett, A. M.; Tour, J. M.; Bard, A. J. J. Am. Chem. Soc. 2002, 124, 5550. (c) Jones, L.; Schumm, J. S.; Tour, J. M. J. Org. Chem. 1997, 62, 1388.

3. See, such as: (a) Nilsson, D.; Watcharinyanon, S.; Eng, M.; Li, L.; Moons, E.; Johansson, L. S. O.; Zharnikov, M.; Shaporenko, A.; Albinsson, B.; Mårtensson, J. Langmuir 2007, 23, 6170. (b) Marx, E.; Chiesa, M.; Borg, M.; Bengtsson, S.; Less, R. J.; Raithby, P. R.; Ford, C. J. B.; Greenham, N. C. Appl. Phys. Lett. 2006, 88, 143107. (c) Fan, F. F.; Yao, Y.; Cai, L.; Cheng, L.; Tour, J. M.; Bard, A. J. J. Am. Chem. Soc. 2004, 126, 4035. (d) Cheng, L.; Yang, J.; Yao, Y.; Price, D. W.; Dirk, S. M.; Tour, J. M. Langmuir 2004, 20, 1335.

4. (a) Xiao, X.; Nagahara, L. A.; Rawlett, A. M.; Tao, N. J. Am. Chem. Soc. 2005, 127, 9235. (b) Tour, J. M.; Cheng, L.; Nackashi, D. P.; Yao, Y.; Flatt, A. K.; Angelo, S. K. S.; Mallouk, T. E.; Franzon, P. D. J. Am. Chem. Soc. 2003, 125, 13279. (c) Tour, J. M.; Rawlett, A. M.; Kozaki, M.; Yao, Y.; Jagessar, R. C.; Dirk, S. M.; Price, D. W.; Reed, M. A.; Zhou, C. W.; 
Chen, J.; Wang, W.; Campbell, I. Chem. Eur. J. 2001, 7 (23), 5118. (d) Chen, J.; Wang, W.; M. A. Reed, M. A.; Rawlett, A. M.; Price, D. W.; Tour, J. M. Appl. Phys. Lett. 2000, 77 (8), 1224. (e) Chen, J.; Reed, M. A.; Rawlett, A. M.; Tour, J. M. Science 1999, 286, 1550.

5. See, such as: (a) Fan, F. F.; Lai, R. Y.; Cornil, J.; Karzazi, Y.; Brédas, J.; Cai, L.; Cheng, L.; Yao, Y.; Price, D. W.; Dirk, S. M.; Tour, J. M.; Bard, A. J. J. Am. Chem. Soc. 2004, 126, 2568. (b) Majumdar, N.; Gergel-hackett, N.; Bean, J. C.; Harriott, L. R.; Pattanaik, G.; Zangari, G.; Yao, Y.; Tour, J. M. J. Electron. Mater. 2006, 35 (1), 140. (c) Majumder, C.; Mizuseki, H.; Kawazoe, Y. J. Mol. Struct. THEOCHEM 2004, 681, 65. (d) Seminario, J. M.; Zacarias, A. G.; Tour, J. M. J. Am. Chem. Soc. 2000, 122 (13), 3015.

6. (a) Tour, J. M. Molecular Electronics: Commercial Insights, Chemistry, Devices, Architecture and Programming. World Scientific: Singapore, 2003. (b) Wang, C.; Bryce, M. R.; Gigon, J.; Ashwell, G. J.; Grace, I.; Lambert, C. J. J. Org. Chem. 2008, 73, 4810. (c) Weibel, N.; Błaszczyk, A.; Hänisch, C.; Mayor, M.; Pobelov, I.; Wandlowski, T.; Chen, F.; Tao, N. Eur. J. Org. Chem. 2008, 136.(d) Wang, C.; Batsanov, A. S.; Bryce, M. R.; Sage, I. Synthesis 2003, 13, 2089.

7. See, such as: (a) Price, D. W. Dirk, J. S. M.; Maya, F.; Tour, J. M. Tetrahedron 2003, 59, 2497. (b) Dirk, S. M.; Price, D. W.; Chanteau, S.; Kosynkin, D. V.; Tour, J. M. Tetrahedron 2001, 57, 5109. (c) Maya, F.; Tour, J. M. Tetrahedron 2004, 60, 81. (d) Price, D. W.; Tour, J. M. Tetrahedron 2003, 59, 3131. (e) Maya, F.; Chanteau, S. H.; Cheng, L.; Stewart, M. P.; Tour, J. M. Chem. Mater. 2005, 17, 1331. (f) Flatt, A. K.; Dirk, S. M.; Henderson, J. C.; Shen, D. E.; Su, J.; Reed, M. A.; Tour, J. M. Tetrahedron 2003, 59, 8555. (g) Flatt, A. K.; Yao, Y.; Maya, F.; Tour, J. M. J. Org. Chem. 2004, 69, 1752. (h) Yu, B. C.; Shirai, Y.; Tour, J. M. Tetrahedron 2006, 62, 10303.

8. (a) Negishi, E.; Anastasia, L. Chem. Rev. 2003, 103, 1979. (b) Sonogashira, K.; Tohda, Y.; Hagihara, N. Tetrahedron lett. 1975, 4467. (c) Meier, H.; Mühling, B. Arkivoc 2009, (ix), 57. (d) Bang, H.; Han, S. Y.; Choi, D. H.; Hwang, J. W.; Jun, J. G. Arkivoc 2009, (ii), 112. (e) Cho, J.; Zhao, Y.; Tykwinski, R. R. Arkivoc 2005, (iv), 142. (f) Martins, M. A. P.; Rossatto, M.; Rosa, F. A.; Machado, P.; Zanatta, N.; Bonacorso, H. G. Arkivoc 2007, (i), 205.

9. (a) Miyaura, N.; Suzuki, A. Chem. Rev. 1995, 95, 2457. (b) Nicolaou, K. C.; Bulger, P. G.; Sarlah, D. Angew. Chem. Int. Ed. 2005, 44, 4442. (c) Kotha, S.; Lahiri, K. Eur. J. Org. Chem. 2007, 1221. (d) Ji, H. Y.; Zhang, Z.; An, Y.; Fu, X. L.; Yang, Y. Gao, G. H. Arkivoc 2009, (xi), 11. (e) Özdemir, I.; Demir, S.; Çetinkaya, B. Arkivoc 2007, (xiii), 71. (f) Mukherjee, A.; Sarkar, A. Arkivoc 2003, (ix), 87. (g) Wu, Y.; Yang, L.; Zhang, J.; Wang, M.; Zhao, L.; Song, M.; Gong, J. Arkivoc 2004, (ix), 111.

10. Błaszczyk, A.; Chadim, M.; Hänisch, C.; Mayor, M. Eur. J. Org. Chem. 2006, 3809.

11. Taher, D.; Walfort, B.; Lang, H. Inorg. Chim. Acta 2006, 359 (6), 1899.

12. Weber, H. B.; Reichert, J.; Weigend, F.; Ochs, R.; Beckmann, D.; Mayor, M.; Ahlrichs, R.; Lohneysen, H. v. Chem. Phys. 2002, 281, 113.

13. Price, D. W.; Dirk, S. M.; Rawlett, A. M.; Chen, J.; Wang, W.; Reed, M. A.; Zacarias, A. G.; Seminario, J. M.; Tour, J. M. Mater. Res. Soc. Symp. Proc. 2001, 660, JJ941/D741. 
